Preface

This historical review on River Blindness, onchocerciasis, is written in honour of (i) the 120-year anniversary of the Bernhard Nocht Institute for Tropical Medicine (BNITM), founded in 1900 in Hamburg in the sequel of a tremendous cholera outbreak that hit Hamburg in 1892, (ii) the 75-year anniversaries of Acta Tropica and (iii) the Swiss Tropical and Public Health Institute (Swiss TPH), both founded in 1944 in Basel by the renowned scientist of tropical medicine Rudolf Geigy (1902-1995). Geigy was the first director of the former Swiss Tropical Institute (STI) and simultaneously the first editor of Acta Tropica.

River Blindness caused by the tissue filaria Onchocerca volvulus was one focus of research in the BNITM over almost 60 years documented in about 300 publications. Consequently, in this historical review article we have cited major articles based on research conducted at the BNITM.

In addition to this historical review article on onchocerciasis two historical articles on 75 year of both, Acta Tropica and Swiss TPH, are jointly published in in one issue of Acta Tropica.

\title{
Review
}

\section{Onchocerciasis (River Blindness) - more than a Century of Research and Control}

\author{
Norbert W, Brattig ${ }^{1 *}$, Robert A. Cheke ${ }^{2}$, Rolf Garms ${ }^{1}$ \\ ${ }^{1}$ Bernhard Nocht Institute for Tropical Medicine, Hamburg, Germany, \\ ${ }^{2}$ Natural Resources Institute, University of Greenwich, Kent, UK \\ *corresponding author
}

\section{Abstract}

This review summarises more than a century of research on onchocerciasis, also known as river blindness, and its control. River blindness is an infection caused by the tissue filaria Onchocerca volvulus affecting the skin, subcutaneous tissue and eyes and leading to blindness in a minority of infected persons. The parasite is transmitted by its intermediate hosts Simulium spp. which breed in rivers. Featured are history and milestones in onchocerciasis research and control, state-of-the-art data on the parasite, its endobacteria Wolbachia, on the vectors, previous and current prevalence of the infection, its diagnostics, the interaction between the parasite and its host, immune responses and the pathology of onchocerciasis. Detailed information is documented on the time course of control programmes in the afflicted countries in Africa and the Americas, a long road from previous programmes to current successes in control of the transmission of this infectious disease. By development, adjustment and optimization of the control measures, transmission by the vector has been interrupted in foci of countries in the Americas, in Uganda, in Sudan and elsewhere, followed by onchocerciasis eliminations. The current state and future perspectives for control, elimination and eradication within the next 20-30 years are described and discussed. This review contributes to a deeper comprehension of this disease by a tissue-dwelling filaria and it will be helpful in efforts to control and eliminate other filarial infections. 


\section{Introduction and history}

Onchocerciasis - commonly known as river blindness - is an infectious disease caused by the parasitic filaria Onchocerca volvulus Leuckart 1893 (Parsons, 1908) belonging to the tissue-residing nematodes (class Chromadorea). The name Onchocerca derived from a combination of the Greek words 'onchos' meaning 'hook' and 'kerkos' meaning 'tail'. The infection occurs primarily in sub-Saharan Africa, but it is also found in Yemen and until recently in foci of six countries within Central America (WHO, 1995; WHO 2020 fact sheets, 2019; WHO, 2020). The Global Burden of Disease Study 2010 (Hotez et al., 2014) quantified the burden of almost 300 diseases, including onchocerciasis, in terms of their relative impacts as disability-adjusted life years (DALYs) which was noted as 490,000 for onchocerciasis with 30 million infected people.

Primarily, the infection affects the skin and eyes and the pathology is caused by subcutaneous larvae (microfilariae). Microfilariae were first discovered in 1874 - almost 150 years ago - by John O'Neill, a British naval surgeon in the Gold Coast (Ghana), while examining skin-snips from so-called craw-craw patients suffering from intense acute dermatitis (O'Neill, 1875). Patrick Manson in 1890 first identified the adult microfilariae-releasing worms and in 1893 Rudolf Leuckart described their morphology from subcutaneous infestations as "Filaria volvuloxus", now known as Onchocerca volvulus (Leuckart 1893; Fülleborn, 1908). The genus name Onchocerca had been given to filarial worms infecting cattle by Diesing in 1841. In 1917 Rodolfo Robles (Robles, 1917) published details of the association of dermatitis with subcutaneous nodules, microfilariae and anterior ocular lesions based on research in Guatemala and named the causative worms O. caecutiens ("blinding”) (Brumpt, 1919; Fülleborn, 1924). In 1927, while working in Sierra Leone, Blacklock discovered that blackflies, Simulium (Edwardsellum) damnosum Theobald transmitted O. volvulus causing onchocerciasis (Blacklock, 1927).

Chromosomes of Simulium were first described in 1937 (Painter et al., 1937) and those of the vectors in 1975 (Vajime and Dunbar, 1975), whereas for Onchocerca they were described later (Hirai et al., 1987; Post et al., 1989). The complete genome of O. volvulus was first published in 2016 by Choi et al. (2016) and Cotton et al. (2016). Rickettsia-like endobacteria, Wolbachia, were first reported in Onchocerca in 1977 by Kozek and Figueroa-Marroquin (1977) and the genome of Wolbachia was described in 1999 by Bandi et al. (1999) and Slatko et al. (1999).

A control programme in 11 West African countries applying vector control began with the Onchocerciasis Control Programme (OCP) in 1974 and ended in 2002 (see section 4.2.2.). The OCP started with vector control by insecticide and was expanded to include treatment of Onchocerca-infected people with the microfilaricide ivermectin in 1987. Treatment studies with doxycycline killing the Wolbachia endobacteria (Fig. 10) started in 2003 after initial in vitro experiments in 2000 (Hoerauf et al., 2000, 2001; Abegunde et al., 2016). Crump et al. (2012) described the onchocerciasis chronicle in detail and Table 1 summarises major milestones in the history of onchocerciasis research and control. 


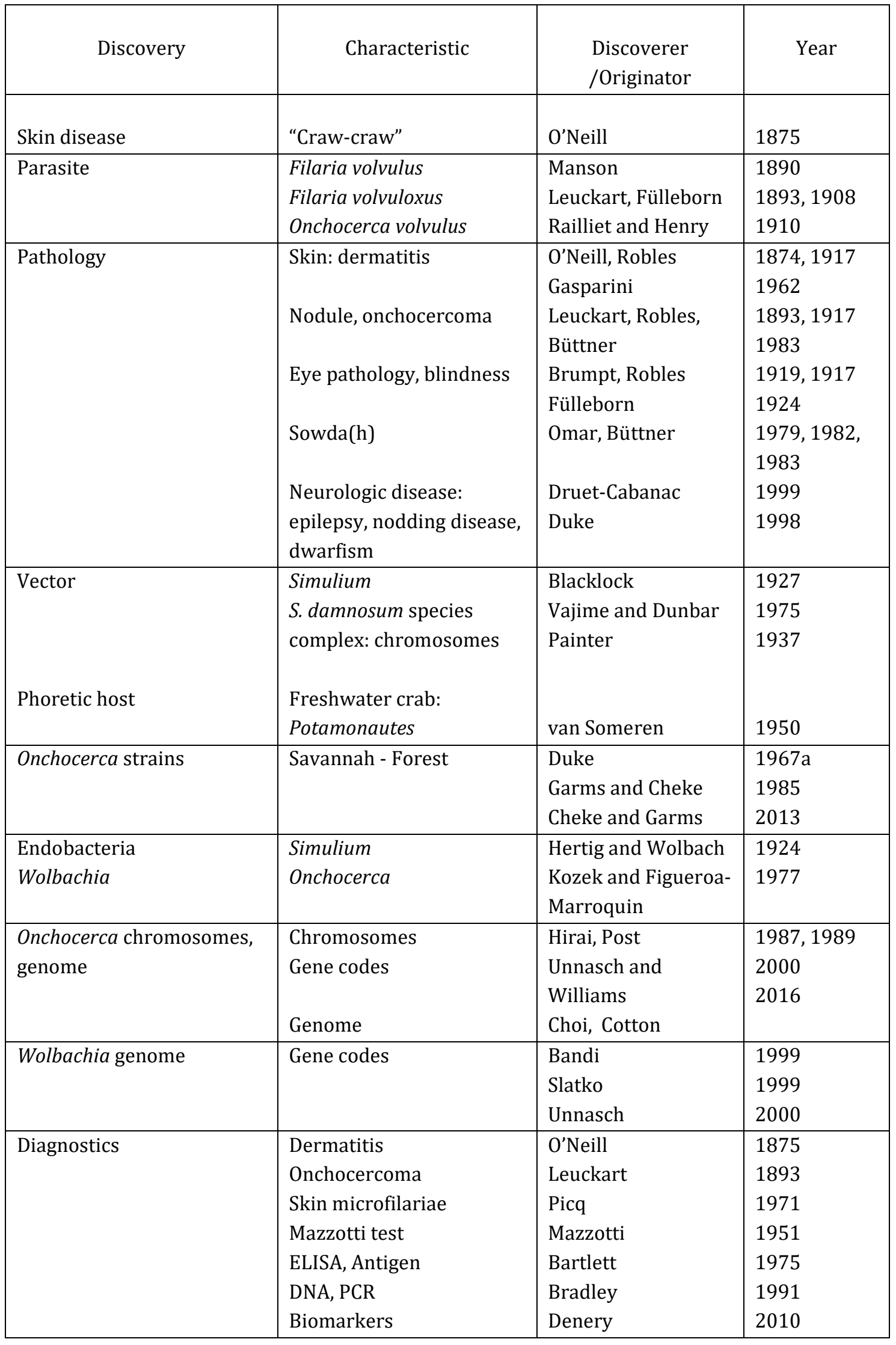




\section{Parasite Onchocerca \\ 2.1. 0 . volvulus life cycle}

\subsubsection{Development of the filaria in the human host}

The infective third stage larvae (L3) of the parasite with a length of 600-700 $\mu \mathrm{m}$ invade the skin when an 0 . volvulus-infected blackfly bites the human host for blood (Fig. 1; Fig. 2). The L3 larvae moult in the skin of the human host to fourth stage larvae which migrate in subcutaneous tissue and grow to the adult female and male stages in 6-12 months. The adult females measure $30-60 \mathrm{~cm}$, while the males are only 1.5-4.5 cm long (Fig. 2). The host reaction against the parasites leads to the formation of nodules known as onchocercomata. Interestingly, the males of the cattle parasite $O$. ochengi, and probably those of $O$. volvulus too, migrate between the nodules, thus from female to female.

In a single day an inseminated adult female can release 1000-3000 microfilariae responsible for the symptoms of the disease. The parasite has an enormous reproductive capacity resulting in millions of microfilariae released from fertilized females during their lives. Schulz-Key and Karam (1986) calculated that the number of microfilariae released from one female during its life of 10-15 years was $>10$ million. The severity of the disease increases with the parasitic load and there is a direct relation between $O$. volvulus microfilarial load and host ocular morbidity (Little et al., 2004). The microfilariae leave the onchocercomata and move through the subcutaneous tissue to reach the cutis where they leave the host if taken up by a vector (Fig. 1).

\subsubsection{Transmission by the vector Simulium}

\subsubsection{The genus Simulium}

There are 2310 living species of blackflies in the family Simuliidae (order Diptera) and 18 known fossil taxa (Adler, 2019). Most species are of no economic importance but 1.5\% of them are vectors of human pathogens causing onchocerciasis and mansonellosis (caused by Mansonella ozzardi in Brazil). Blackflies also transmit Onchocerca spp. to other vertebrates including cattle and, through their biting, they may also cause serious allergic reactions in cattle which can be fatal. In addition, they transmit protozoa to birds including Leucocytozoon spp. causing infections that are sometimes of economic importance for poultry. The major vectors of human onchocerciasis in Africa are members of the $S$. damnosum species complex of which at least 65 different forms have been described, with at least 15 of them acting as vectors (Adler et al., 2010).

In addition to their importance as vectors, blackflies can be serious biting pests of man and animals in both tropical and temperate climates, with examples including S. erythrocephalum in central Europe and S. posticatum in England. Particularly infamous was the Golubac fly S. colombaschense which in 1923 killed 22000 animals (sheep, goats, cattle) in the Danube valley in Yugoslavia, Hungary and Romania (Crosskey, 1990). 
Members of the genus Simulium (Diptera: Simuliidae) have aquatic immature stages. The adults lay their eggs on trailing vegetation or rocks in streams or fast-flowing sections of rivers. Larvae hatch from the eggs and pass through 6 to 11 instars before becoming pupae from which the adults later emerge (Fig. 3). The males do not blood-feed but may obtain sugar-feeds from plants. Most female Simulium are haematophagous and it is this habit which links them to vectorial importance.

In Africa, the most important vectors are members of the S. damnosum species complex, but the disease is also transmitted in East Africa by members of the S. neavei complex (subgenus Lewisellum, 9 species) which has its immature stages phoretic on freshwater crabs. The most important vectors within the $S$. neavei complex are $S$. neavei in Uganda and $S$. woodi in Tanzania. In the Congo $S$. albivirgulatum is a vector in the central basin region (Fain et al., 1981). Recently S. dentulosum and S. vorax have been found biting man in the absence of S. damnosum s.l., S. neavei s.l. and S. albivirgulatum in the Ituri-Albert focus of the Democratic Republic of Congo. Furthermore, DNA tests confirmed the presence of $O$. volvulus in both $S$. dentulosum and S. vorax but few samples of the latter were available and none were infective. In contrast, $30 \%$ of 155 S. dentulosum were infected and $11 \%$ were infective, so a new vector species has been confirmed (R.J. Post et al. unpubl. and pers. comm.).

A variety of vectors is or was responsible for transmission in central and southern America. Members of the S. ochraceum and S. metallicum complexes were involved in Mexico, with the former most active in transmission in Guatemala (Garms and Ochoa, 1979a) before elimination of the disease in that country (Rodríguez-Pérez et al., 2015). In the remaining Amazonas focus that straddles the Venezuela-Brazil border the main vectors are members of the $S$. oyapockense and $S$. guianense species complexes and $S$. incrustatum (Shelley et al., 2010).

\subsubsection{Development of the filaria in Simulium}

The mouthparts of the female vectors of onchocerciasis do not penetrate the skin and absorb blood in the manner of mosquitoes but rather they use their mandibles to rasp at the skin until bleeding occurs and then the blood is lapped up from the resultant pool, hence they are known as pool-feeders (Fig. 3). Any $O$. volvulus microfilariae (Fig. 2.1) that are within the pool may be ingested by the blackflies. In vectors with pronounced buccopharangeal armatures such as members of the $S$. ochraceum complex, many microfilariae may be killed at this stage (Omar and Garms, 1975). In contrast, S. metallicum complex members lack such structures and if they ingest many microfilariae the flies may soon die shortly after their blood-meals (Omar and Garms, 1977), although this does not appear to happen with the $S$. damnosum complex which also lacks marked buccopharangeal armatures.

Once within the stomach with the blood meal the microfilariae must try to escape through the stomach wall into the haemocoel (Fig. 1) before being trapped within the peritrophic matrix that can form within 30 minutes. Bain et al. (1976) suggested that the reason that forest $S$. damnosum harbour higher numbers of developing $O$. volvulus larvae than do savannah forms was because of differences between the 
151

152

153

154

155

156

157

158

159

160

161

162

163

164

165

166

167

168

169

170

171

172

173

174

175

176

177

178

179

180

181

182

183

184

185

186

two vector groups in the structure and speed of development of the peritrophic matrix, with forest flies' membranes being less well sealed and slower to form.

Those microfilariae that reach the haemocoel move into the thoracic muscles, changing their shape to become "sausage stage" forms. These moult to the second stage (L2) and then moult again within the thoraces to become third stage larvae (L3) (Fig. 2.3) before emerging into the female's body cavity to become elongated infective L3 stage larvae that will be capable of infecting a new host (Fig. 1, Fig. 2.3). L3s penetrate several fly organs, the ovaries, the brain, antennae, and the palps, thereby debilitating the fly resulting sometimes in only about $1 \%$ of the flies carrying L3s surviving, according to results of a laboratory study involving S. yahense (Trpis et al., 2006). Because the development process of the Simulium stages takes 6-9 days and the gonotrophic cycle of the females is 2-4 days, it is usually only at their third bite that the flies can transmit infections.

Much of what is known about the transmission of onchocerciasis by different S. damnosum complex members was derived from research conducted during the WHO Onchocerciasis Control Programme in West Africa (OCP). As mentioned in a later section (see section 4.2.2.), the OCP was affected by reinvasions of controlled zones by immigrant flies bred outside the treated areas. Thus, it became important to identify which members of the S. damnosum complex were responsible in order to be able to locate and treat the sources. Garms (1978) showed that savannah species could be separated from forest species by a combination of the colour of the basal wing tufts and the ratio between the lengths of the thoraces and antennae, with the latter being longer and less compressed in forest forms for a given fly size. Later morphological studies (Garms et al., 1982; Garms and Zillmann, 1984; Garms and Cheke, 1985; Meredith et al., 1983)-allowed more of the species, or at least species groups, occurring in the OCP to be identified such that it was possible to compare their transmission abilities (Cheke and Garms, 2013). In general, forest forms carry more 0 . volvulus larvae per infected fly than the savannah forms.

\subsection{Endobacterium Wolbachia}

\subsubsection{Wolbachia in the parasite Onchocerca}

In 1977 Kozek and Figueroa-Marroquin (1977) found intracytoplasmic Rickettsia-like bacteria in $O$. volvulus and Sironi et al. (1995) provided molecular evidence for a close relative of the arthropod endosymbiont Wolbachia in a filarial worm. Rickettsia-like gram-negative endobacteria, alpha 2 proteobacteria, were originally detected in arthropods in 1924 by Hertig and Wolbach (1924), representing the eponym Wolbachia designated in 1936.

Wolbachia are found in the hypodermal cells of the lateral cords of adult filariae and in embryonic stages (Taylor and Hoerauf, 1999, Hoerauf et al., 2000) (Fig. 2.6). The absence of Wolbachia appears to be an ancestral condition (Bandi et al., 2001). The Wolbachia were shown to be mutualistic in Onchocerca (Comandatore et al., 2015), with the endobacteriae appearing to provide essential metabolites to the 
filaria, which contribute to its reproduction and larval development, promoting fertility, embryogenesis and viability of the filaria (Comandatore et al., 2015).

Products of the endosymbionts have been implicated in the pathogenesis of ocular onchocerciasis

190 (Saint André et al., 2002). Recent data support the hypothesis that suspected differences between severe

191 and mild strains of $O$. volvulus (see section 2.3.) may be a function of their relative Wolbachia burden

192 indicating that Wolbachia products may play a central role in the pathogenesis of ocular onchocerciasis

193 (Pearlman, 2003).

194 The genome of onchocercal Wolbachia $(w O v)$ has been analysed (Unnasch and Williams, 2000; Choi et

195 al., 2016). The $956 \mathrm{~kb}$ genome contains 785 predicted protein-coding genes

196 (http://exon.niaid.nih.gov/transcriptome/O_volvulus/v245/wOv_web/wOv_Web.xlsx) including the most

197 abundant proteins: Wolbachia surface protein (WSP, w0v00566) and the chaperone DnaK (w0v00687)

198 (Choi et al., 2016). The proteins were mapped to functional categories, with the top five functions being (i)

199 translation, ribosomal structure, and biogenesis; (ii) post-translational modification, protein turnover,

200 and chaperone; (iii) energy production and conversion; (iv) coenzyme metabolism and cell envelope biogenesis, and (v) outer membrane proteins (Bennuru et al., 2016).

The bacteria in the filariae represent a target for antibiotic therapy (D.W. Büttner, 1997, pers. comm.; Hoerauf et al., 2000, 2002; Taylor et al., 2001). Immunological studies revealed that the hosting endobacteria contribute to inflammatory reactions of the human host of the filariae (Pearlman, 2003).

\subsubsection{Wolbachia in the vector Simulium}

Yen (1975) first reported on intracellular Wolbachia in insects, long before the Wolbachia were identified as intracellular bacteria in filariae. Wolbachia are responsible for cytoplasmic incompatibility in Culex pipiens and numerous subsequent studies revealed that these endobacteria manipulate the reproduction of their arthropod hosts and can move horizontally across species' boundaries. Meanwhile

211 Wolbachia have been demonstrated in numerous mosquito vector species of medical and veterinary

212 importance and have been used to control transmission of dengue fever by releasing Aedes aegypti vectors 213 infected with the wMel strain of Wolbachia (Hoffmann et al., 2011).

214 The presence of Wolbachia in onchocerciasis vectors was first demonstrated by Crainey et al. (2010)

215 who found the endosymbiont in larval samples of Simulium from Ghana. It is unclear if all individuals are

216 infected, as the Wolbachia were found in less than a quarter of specimens, but they have also been

217 detected in S. squamosum and S. yahense adults and in S. oyapockense s.l., with those in the latter differing

218 markedly from the types found in the S. damnosum complex (J.L. Crainey, 2019, pers. comm). If there are

219 consistent interspecific differences in the frequencies of occurrence and/or the varieties present in

220 different vectors, it is possible, given Wolbachia's known manipulation of reproductive capacities and

221 vector status in other arthropods, that they could be of epidemiological importance, e.g. by accounting for

222 differences in parasite burdens between forest and savannah vectors in West Africa. Wolbachia may also 
be implicated in why Simulium are so difficult to colonise in the laboratory, e.g. by male-killing, and this

224 hurdle will need to be overcome if the potential of using Wolbachia in Simulium control can be realised.

225 Nevertheless, progress towards such a goal has been made with the discovery of a prophage element

226 within S. squamosum E that includes a SpvB-like protein at the extreme terminal end of its sequence

227 which is suspected of having insecticidal properties (Crainey et al., 2017).

\subsection{Phylogeny and biology of Onchocerca}

The phylogenetic tree of the genus Onchocerca Leuckart comprises 14 species divided into three clades (Lefoulon et al., 2017). The third clade is composed of $O$. volvulus and the related species $O$. ochengi, O. gibsoni and $O$. gutturosa which parasitize domesticated bovids. O. volvulus is genetically most closely related to $O$. ochengi (https://parasite.wormbase.org/Onchocerca_ochengi_prjeb1465/Info/Index). The $O$. volvulus / O. ochengi sister relationship supports the scenario that the human parasite resulted from a host transfer by the bovine 0 . ochengi, or its ancestor (Bain, 2002; Morales-Hojas et al., 2006), possibly during the course of cattle domestication and hence within the last 10,000 years, with $O$. ochengi switching into humans to become $O$. volvulus (Lefoulon et al., 2017).

On the basis of the genetic similarity of $O$. volvulus and $O$. ochengi, the bovine infection by $O$. ochengi has become famous as a natural model or 'analogue' of human onchocerciasis (Trees et al., 2000; Makepeace and Tanya, 2016). A multitude of experimental joint studies by the University of Ngaoundéré and the University of Tübingen, Germany, demonstrated similarities in the stage-specific proteome (Armstrong et al., 2016), in excretory-secretory (E/S) peptides (Eberle et al., 2015), in immune recognition of ES proteins (Manchang et al., 2015), in cross-protection (Wahl et al., 1998), in crossvaccination (Achukqui et al., 2007), and in the conserved nature of circulating miRNA (Quintana et al., 2015). Further, similarities in the immune antigen recognition pattern was reported in $O$. ochengi-infected cattle and in an O. volvulus-infected chimpanzee (Graham et al., 2000) confirming the use of the primate as a surrogate host for Onchocerca infection. The comparability of both infections, nevertheless, appears to be limited since in the evolutionarily primordial 0 . ochengi infections the parasite is highly adapted to its bovine host eliciting minimal pathology, whereas in the evolutionarily younger parasitism by 0 . volvulus severe damage often results (see section 3.3.).

251 (https://pubmed.ncbi.nlm.nih.gov/27869790/?from_sort=pubdate\&from_term=Cotton+JA\&from_cauthor _id $=27881553 \&$ from_pos $=4$ ).

Differences between the manifestation of onchocerciasis in forest and savannah regions, in particular between blinding rates, gave rise to a two-strain hypothesis (Duke et al. 1966). Analyses of entomological data (Cheke and Garms, 2013) and a reanalysis of pre-control blindness data (Cheke et al., 2020) have led to this hypothesis being questioned. Although some molecular studies of different $O$. volvulus populations revealed differences between savannah and forest strains, for instance by being distinguishable using the 
(Morales-Hojas et al., 2007). Recent studies involving nuclear DNA have not confirmed clear cut distinctions but did show that parasites from the two zones can and do interbreed (Choi et al., 2016).

The complete genome of the mitochondria of $O$. volvulus

262 (https://parasite.wormbase.org/Onchocerca_volvulus_prjeb513/Info/Index) was published by Crainey et 263 al. (2016) while the total genome of $O$. volvulus comprises a $97 \mathrm{Mb}$ nuclear genome coding 12,143 protein264 coding genes, and the onchocercal Wolbachia have a $956 \mathrm{~kb}$ genome containing 785 predicted protein265 coding genes (Cotton et al., 2016; http://parasite.wormbase.org/Onchocerca_volvulus_prjeb513/Info/Index; 266 http://exon.niaid.nih.gov/transcriptome/O_volvulus/v245/wOv_web/wOv_Web.xlsx). Nine percent of the 267 genes are O. volvulus-specific (Unnasch et al., 2000; Cotton et al., 2016; Choi et al., 2016). Recent reports 268 on the transcriptome and proteome of 0 . volvulus and its Wolbachia endosymbiont (Bennuru et al., 2016) 269 open up candidate molecules for diagnosis, new biomarkers, vaccine and drug targets. Further, the proteome of $O$. volvulus identified various mimics and antagonists of human cytokines and chemokines. Furthermore, the genome encodes numerous serine protease inhibitors such as serpins, as well as cysteine protease inhibitors like cystatin (Cotton et al., 2016). These proteins can interfere with antigen processing and presentation indicating parasite interference with host immune responses, thereby facilitating and promoting their survival in immunocompetent hosts. Interestingly, distinct encoded proteins are similar to human autoantigens, which may be implicated in the pathogenesis of eye diseases and nodding syndrome.

Onchocerciasis is very probably an anthroponosis. Humans are almost certainly the unique host of $O$. volvulus - although there are two reports indicating that primates can also host $O$. volvulus. Caballero and Barrera (1958) reported recovery of a nodule containing fertile 0 . volvulus adults from a golden spider monkey (Ateles geoffroyi) captured in Chiapas (Mexico), and a natural infection with 0 . volvulus has been found in a gorilla (Gorilla gorilla) by van den Berghe et al. (1964) in the Congo. Neumann et al. (1964) reported experimental onchocercal ocular lesions in chimpanzees (Pan troglodytes) and these primates were used as surrogate hosts in experimental infections for a long period to investigate humoral and cellmediated immune responses, vaccination and drug effects (Greene, 1987; Taylor et al., 1988; Soboslay et al., 1991; Prince et al., 1992) until 2013 when the USA's National Institutes of Health (NIH) banned invasive research on chimpanzees (Knight, 2008).

\subsection{Prevalence}

Estimates of prevalence vary substantially. Thus, the WHO stated for 1983 that globally 85.5 million people were at risk and about 37 million people were infected with 0 . volvulus (Amazigo et al., 2008), with 0.34 million of the infected blinded by onchocerciasis (WHO, 1987). For 1995, the WHO noted 123 million 
Onchocerciasis is almost exclusively (>99\%) prevalent in 31 countries of sub-Saharan Africa, but about 20,000 infected persons live in Yemen (Büttner et al., 1982; Connor et al., 1983; WHO 2019). In terms of populations living in areas where more than $50 \%$ live in areas where the predicted nodule prevalence is greater than 20\%, the main countries are the Democratic Republic of Congo with 23.3 million people,

300 Nigeria (14.3 million), Ethiopia (5.9 million) and Cameroon (5.2 million)(Zouré et al., 2014). In central

301 Africa - the Central African Republic, Gabon, Democratic Republic of Congo, Angola, Sudan, Ethiopia,

302 Uganda, Nigeria, Cameroon - onchocerciasis is co-endemic with loiasis. Loiasis, caused by the filaria Loa 303 loa and transmitted by horseflies (Chrysops dimidiatus and C. silaceus) affect the eyes (African eye worm) 304 (Vinkeles Melchers et al., 2020). Loiasis coinfection is a major concern for onchocerciasis elimination in 305 Africa (see 4.1.).

Onchocerciasis also occurred in 13 isolated foci in six countries of Latin America, infecting 97200 people with 500,000 people at risk (Sauerbrey, 2018; CDC 2013). Active transmission currently is limited to two foci among Yanomami indigenes in adjacent border areas of Venezuela and Brazil (CDC, 2013) (see section 4.3.3.). The fact that $O$. volvulus populations of African savannah and Central America are genetically indistinguishable, indicates that onchocerciasis was introduced into America by the entry of infected Africans from the savannah (Crump et al., 2012).

\subsection{Diagnostics}

Tests for diagnosing onchocerciasis are summarised in Table 2. Onchocerciasis is primarily diagnosed clinically by detecting onchodermatitis, by subcutaneous nodules (onchocercomata), often located at the hips, and by detection of microfilariae in skin snips (microfilaridermia) requiring a microscopic examination (Picq et al., 1971; Albiez et al., 1988a; Alhassan et al., 2016). Skin patch testing (Mazzotti reaction) with diethylcarbamazine (DEC) can indicate microfilaridermia (Mazzotti, 1951). Awadzi et al.

320 (2015) reported a clinical evaluation of a transdermal delivery technology-based patch for the diagnosis of $O$. volvulus infection via skin exposure to diethylcarbamazine. Ocular status can also help diagnosis, if pathological findings such as punctate keratitis or even microfilariae in the anterior chamber are noted 323 (O’Day and Mackenzie, 1985).

Laboratory analyses have been used since 1975 (Bartlett et al., 1975) to detect serum antibodies against Onchocerca surface proteins, preferentially IgG4, in infected persons by applying enzyme-linked 326 immunosorbent assays (ELISA), by a fluorescent antibody staining technique or more recently by rapid327 format antibody card test (Bartlett et al., 1975; Weil et al., 2000). As target antigens numerous O. volvulus328 specific proteins have been investigated, in particular low molecular weight antigens such as Ov16 in 329 addition to numerous additional proteins or hybrid proteins (Ov33, Ov10, Ov20 (Ov-FAR-1), Ov-RAL-2, 330 Ov7, OvSOD1, Ov-ENO, Ov103, Ov9.3, OvMSA-1) (Lucius et al., 1988; Lobos et al., 1991; Mpagi et al., 2000; 331 Andrews et al., 2008; McNulty et al., 2015; Unnasch et al., 2018). However, the sensitivity and species 
specificity of antigen recognition assays are mostly of limited value because of cross-reactions with

333 proteins from other filariae.

334 Ov16 was identified as highly diagnostic, mostly applied antigen (Lobos et al., 1991; Denery 2010;

335 Lont et al., 2017; Bennuru et al., 2020). Subsequently, peptide epitopes (OvMP-23, OvNMP-48; OvOC9384,

336 OvOC198, and OvOC5528; rOVOC10469 and rOVOC3261) (Gonzalez-Moa, 2018; Lagatie et al., 2019;

337 Bennuru et al., 2018) were applied in ELISA analysis verifying the sensitivity and specificity by receiver

338 operating characteristic (ROC) analysis. The lateral flow rapid assay with 0v16 and rOVOC3261 was

339 developed as the best antigen-antibody test with $94 \%$ sensitivity and applied as the current diagnostic

340 tool to verify interruption of transmission of $O$. volvulus (Vlaminck et al., 2015; Unnasch et al., 2018).

341 Some molecular biological analyses with high specificity have been developed such as real time

342 polymerase chain reaction RT-PCR and colorimetric loop-mediated isothermal amplification (LAMP,

343 Alhassan, 2016). The amplification assay targets a repeated $O$. volvulus sequence 0-150, present in the $O$.

344 volvulus genome with a unit length of roughly $150 \mathrm{bp}$.

345 Recently circulating biomarkers, e.g. peptides (OvOC3261, N-acetyl-tyramine- 0-glucuronide, NATOG)

346 (Globisch et al., 2013) or lipids (phospholipid, glycerophosphorlipid) as well as micro RNA have been

347 developed as promising proof-of-contact diagnostic tests (Quintana et al., 2015; Lagatie et al., 2016;

348 Gonzalez-Moa et al., 2018; Bennuru et al., 2020; Macfarlane et al., 2020). 
Table 2. Diagnostic tests for detection of 0 . volvulus infection

\begin{tabular}{|c|c|c|c|}
\hline Test & Target & Principle & Reference \\
\hline Biopsy & Microfilaria & $\begin{array}{l}\text { Microscopic detection of } \\
\text { microfilariae in a skin snip } \\
\text { biopsy }\end{array}$ & $\begin{array}{l}\text { Picq } 1971 \\
\text { Alhassan } 2016\end{array}$ \\
\hline $\begin{array}{l}\text { Mazzotti patch } \\
\text { test }\end{array}$ & Microfilaria & $\begin{array}{l}\text { Microfilaria-induced } \\
\text { inflammatory reaction by } \\
\text { provocation with DEC }\end{array}$ & $\begin{array}{l}\text { Mazzotti } 1958 \\
\text { Awadzi } 2015\end{array}$ \\
\hline \multirow[t]{3}{*}{$\begin{array}{l}\text { Antibody } \\
\text { detection } \\
\text { (ELISA) }\end{array}$} & $\begin{array}{l}\text { Onchocerca } \\
\text { antigens, hybrid: } \\
\text { Ov16, Ov20 (Ov- } \\
\text { FAR-1), Ov33, } \\
\text { Ov10, Ov7, } \\
\text { Ov103, OvSOD1, } \\
\text { Oc9.3, Ov-MSA-1 }\end{array}$ & $\begin{array}{l}\text { Recognition of surface or } \\
\text { secreted antigens of Onchocerca } \\
\text { by serum IgG (IgG1, IgG4), IgM } \\
\text { in infected humans }\end{array}$ & $\begin{array}{l}\text { Lucius } 1988 \\
\text { Lobos } 1990 \\
\text { Andrews } 2008 \\
\text { Burbela } 2010 \\
\text { McNulty } 2015 \\
\text { Lagatie } 2018 \\
\text { Unnasch } 2018\end{array}$ \\
\hline & $\begin{array}{l}\text { Peptide epitope } \\
\text { OvMP-23, } \\
\text { OvNMP-48 } \\
\text { OvOC9384, } \\
\text { OvOC198, } \\
\text { OvOC5528 }\end{array}$ & Recognition by IgG & $\begin{array}{l}\text { Lagatie } 2019 \\
\text { Gonzalez-Moa } 2018\end{array}$ \\
\hline & $\begin{array}{l}\text { Ov16 and } \\
\text { rov0c3261 }\end{array}$ & $\begin{array}{l}\text { Antigen recognition by a Point- } \\
\text { of-Care lateral flow rapid assay }\end{array}$ & $\begin{array}{l}\text { Steel } 2015 \\
\text { Bennuru } 2020\end{array}$ \\
\hline $\begin{array}{l}\text { qPCR }^{1} \\
\text { LAMP }^{2}\end{array}$ & $\begin{array}{l}\text { Genes: } \\
\text { 0-150 } \\
\text { Cox1 } \\
\text { miRNA }^{3}\end{array}$ & $\begin{array}{l}\text { DNA amplification } \\
\text { qPCR, LAMP }\end{array}$ & $\begin{array}{l}\text { Zimmermann } 1994 \\
\text { Alhassan } 2016 \\
\text { Macfarlane } 2020\end{array}$ \\
\hline $\begin{array}{l}\text { Biomarker } \\
\text { Lateral flow } \\
\text { immunoassay, } \\
\text { Mass } \\
\text { spectrometry }\end{array}$ & $\begin{array}{l}\text { NATOG }^{4} \\
\text { Phospholipids } \\
\text { OvOC3261 } \\
\text { OvOC9384, } \\
\text { OVOC9087, } \\
\text { OvOC835, } \\
\text { OvOC224 }\end{array}$ & $\begin{array}{l}\text { Lateral flow immunoassay, } \\
\text { Liquid chromatography tandem } \\
\text { mass spectrometry method (LC- } \\
\text { MS/MS) }\end{array}$ & $\begin{array}{l}\text { Denery } 2010 \\
\text { Globisch } 2013 \\
\text { Bennuru } 2018 \\
\text { Bennuru } 2020\end{array}$ \\
\hline
\end{tabular}

${ }^{1}$ quantitative polymerase chain reaction, ${ }^{2}$ Loop-mediated isothermal amplification, ${ }^{3}$ microRNA,

354 4NATOG, N-acetyltyramine-O-glucuronide

\subsection{Pathology}

The primary manifestation of an 0 . volvulus infection is itching of the skin. The skin affliction results

359 from the migration of a myriad of microfilariae from the subcutaneous nodules (onchocercomata),

360 harbouring fertilised adult female and male filariae, into the adjacent skin. In the course of the infection an

361 acute papular rash develops into a chronic papular dermatitis which may be associated with

362 lichenification, development of papules, atrophy, and depigmentation. The skin manifestation may 
comprise so-called "leopard, elephant or lizard skin". In addition, oedema and lymphadenopathy can occur and so-called "hanging groins" (Puente et al., 2018).

A distinct variation of skin pathology (Fig. 5) expressing severe chronic onchodermatitis with dark black hyperpigmentation and plaques, designated as sowda(h) (arabic word "aswad" for black) (Fig. 5.3), was originally observed in the Yemen by Gasparini (1962) and subsequently investigated by numerous scientists (Büttner et al., 1982; Connor et al., 1983; Ottesen, 1995; Richard-Lenoble et al., 2001, Al-Kubati et al., 2018). Sowda patients were also found in other endemic countries including Nigeria, Sudan, Ethiopia, Liberia, Guatemala and Ecuador (see section 4.3.1.). Patients with sowda manifestations exhibited generally low densities of microfilariae (Büttner and Racz, 1983; Büttner, 1984; Connor et al, 1983; Richard-Lenoble et al., 2001).

Adult female worms induce an inflammatory response in the infected host and infiltration of immune cells leads to the formation of a granuloma and then a subcutaneous nodule, an onchocercoma (Burchard et al., 1979) (Fig. 5.6). Also, excreted filarial proteolytic, angiogenic and collagen-inducing proteins promote the formation of the nodule or connective tissue-degrading activity (Haffner et al., 1998). The onchocercomata harbour 2-20 fixed and clustering females and 1-10 males migrating from nodule to nodule.

The most aggravating pathology in onchocerciasis is represented by severe visual impairment afflicting 500,000 people and blindness occurring in approximately 270,000 persons rendering river blindness the second most frequent cause of infectious blindness (Albiez et al., 1981; Hall and Pearlman, 1999). Most affected are patients with onchocercomata in the upper part of the body, including the head when microfilariae invade the eyes. The host's reaction to the infiltrated microfilariae initiate corneal opacities or punctate keratitis that can develop into corneal scarring and a sclerosing keratitis.

Rarely, a disfiguring manifestation involving retarded growth (dwarfism, Nakalanga syndrome) occurs in onchocerciasis patients (Duke, 1998). Further pathogenic features are varying neurological diseases, nodding syndrome and epilepsy associated with autoimmunity (Colebunders et al., 2017; Johnson et al., 2017).

\subsection{Parasite- Host interaction}

The parasite Onchocerca, residing and developing in a human host, can survive because its resilient cuticle surface resists the host's efforts to cope with the invading parasite. In addition, the parasite synthesizes and releases a myriad of intercepting excretory/secretory $(E / S)$ molecules, via extracellular vesicles or directly. These molecules include antioxidants, protease-inhibitors, carbohydrate- and lipidbinding molecules and cytokine regulators, which mitigate and detoxify the offending host's components (Hewitson et al., 2009; Njume et al., 2019). O. volvulus microfilariae also release matrix-degrading serine and metalloproteases which can degrade components of the dermal extracellular matrix and elastic fibres 
of host tissue, as observed in chronic onchocerciasis (Haffner et al., 1998). Vital secreted defence compounds of the filaria represent antioxidants like superoxide dismutase, peroxidoxin and thioredoxin peroxidase. Also, proteinase inhibitors, onchocystatin and serpin, are released as protection against host immune attack (Henkle-Dührsen and Kampkötter, 2001; Schönemeyer et al., 2001; Hewitson et al., 2009).

In the course of the infection the host elicits a sequence of defence mechanisms reviewed by Ottesen 404 (1995), Brattig (2004a) and Maizels et al. (2018). In response to the filarial antigens the B-lymphocytes of 405 the host produce antibodies, predominantly immunoglobulin G4 (IgG4) and IgE antibody isotypes (Brattig 406 et al., 1994; Garraud et al, 1996; Adjobimey and Hoerauf, 2010). The blocking IgG4 type antibodies 407 enhance the parasite's potential for host response evasion by inhibiting detrimental reactions since (i) 408 IgG4 represent non-complement-fixing immunoglobulins and (ii) IgG4 cannot induce antibody-dependent 409 cell-mediated cytotoxicity (ADCC) (Adjobimey and Hoerauf, 2010). Hence, high IgG4 concentrations are 410 found in immunosuppressed patients with high microfilarial loads (Adjobemey and Hoerauf, 2010; 411 Ottesen, 1995).

The defence mechanisms also include cellular responses of the adaptive and innate immune system: lymphocyte and granulocyte populations are activated to secrete cytokines and toxic compounds which affect the parasite (Ottesen 1995; Maizels et al. 2018). In Onchocerca-infected individuals the Thelper lymphocyte populations Th1, Th2, Th17 and regulatory T cells (Treg) are stimulated - predominantly occurring as a Th2-response (Brattig et al., 1987; Plier et al., 1995; Timmann et al., 2003; Allen and Sutherland, 2014). The helminth initiated lymphocyte subsets produce the cytokines IL-4, IL-13, IL-5, IL10, and TGF-beta which subsequently initiate multiple reactions from the innate immune system (Turaga et al., 2000; Brattig et al., 1997; 2002; Soboslay et al., 1999; Dötze et al., 2000; Hoerauf and Brattig, 2002).

Characteristic of the innate immune response are eosinophilic and neutrophilic granulocytes, mast cells and alternatively activated macrophages (Brattig, 2004a; Maizels et al., 2018). Predominant eosinophilic granulocytes are activated by IL-5, released from Th2 cells, and their infiltration in the tissue is regulated by IL-4 and IL-13. Such activated eosinophils adhere and degranulate at the surface of microfilariae (Fig. 7.1-2) and infective larvae (Fig. 7.3-4) (Medina-De la Garza et al., 1990; Strote et al., 1990; Brattig et al., 1991; Abraham et al., 2004). Eosinophilic effector cells produce reactive oxygen species and secrete, via extracellular granules, multiple toxic molecules including oxygen radicals, eosinophil peroxidase, major basic proteins, eosinophil cationic proteins , eosinophil-derived neurotoxin and cytokines such as IL-10 and even IL-13 (Tischendorf et al., 1992; Pearlman 1997; Weller and Spencer, 2017). In addition to the eosinophils, mast cells are also operative in the host responses against helminths

430 (Ottesen, 1995; Korten et al. 1998). The Th1-associated response of neutrophilic granulocytes reflect the 431 presence of the endobacteria (see section 3.5.) (Brattig et al., 2001; Tamarozzi et al., 2016). In addition to 432 anti-parasitic reactions, the innate immune system is involved in wound repair mechanisms (Weller and 433 Spencer, 2017). 
These multifarious reactions result in an inflammatory or immunosuppressed status which affect both competitors (Mackenzie et al., 1985; Ottesen, 1995; Brattig, 2004a). Hence, the pathogenesis of onchocerciasis is considered to be a consequence of long-standing reciprocal reactions of both parasite and host. The genetic constitution of the host represents one basic factor determining the variability of the host reactivities (Meyer et al., 1994; Timmann et al., 2008), and the presentation of a spectrum of disease manifestations (Ottesen, 1995, Büttner, 1984; Lucius et al., 1986; Hoerauf et al., 2003a; Brattig, 2004a).

Patients with a hyperreactive form of onchocerciasis, Sowda(h) (see section 3.3.) exhibit a particular host-parasite interaction (Bartlett et al., 1978; Connor et al., 1983; Ottesen, 1995; Brattig, 2004a). The high inflammatory potential visibly manifests in the skin showing hyper-pigmentation, lesions, pruritus and lichenification (Fig. 5). This activated state corresponds with strong cellular immune responses. Characteristic are Th2 and Th17-Th2 lymphocytes and their secreted products such as IL-13, IL-4 and IL17; they are associated with low Treg cells $\left(C D 4{ }^{+} C D 25^{\text {hiFoxp}}{ }^{+}\right)$reactivity (Brattig et al., 1987; Hoerauf et al., 2002; Katawa et al., 2015). Furthermore, high numbers of eosinophlic granulocytes,together with their released toxic cell products, and mast cells occur in hyperreactive onchocerciasis (Medina-De la Garza et al., 1990; Rubio-de Krömer et al., 1995; Tischendorf et al., 1992; Hoerauf et al., 2002). Although only a small number of microfilariae occur in the skin, the lack of Treg cells results in uncontrolled inflammatory responses (Brattig 2004a; Hoerauf and Brattig, 2002; Katawa et al., 2015). Serologically, strongly increased antibody including autoantibody responses are characteristic of sowda patients (Brattig et al., 1994; Gallin et al., 1995) (see section 3.3.). In consequence, these strong immune responses are associated with the reported low level of microfilarial density (Omar et al., 1979; Büttner and Racz., 1982, 1983; Siddiqui and Khawajah, 1991).

One major option for an effective host immune response is a prophylactic vaccine against the parasite, notably that spurred on and advanced by Sarah Lustigman (Lustigman et al., 2002, 2018; Hotez et al., 2015; George et al., 2019). Vaccines are aimed at preventing infection by infective larvae (anti-L3), and/or reducing microfilariae thereby complementing the control or elimination of onchocerciasis. Numerous proteins released by the filariae have been investigated for their vaccine potential; these comprise 0v-103, Ov-RAL-2, Ov-CHI-1, Ov_ALT-1, Ov-B20, Ov28CRP, Ov-GAPDH (Steisslinger et al., 2015; Lagatie et al., 2018; Lustigman et al., 2018). The alum-adjuvanted vaccine consisting of Ov-103, expressed at the surface of microfilariae, and Ov-RAL-2, found in the hypodermis of infective larvae, have the potential of reducing the infection by inhibition of moulting and survival of larvae. The development of cytophilic antibodies against the antigens and of interleukins effect antibody-dependent cellular cytotoxicity (George et al., 2019). Further, an immunomics approach with serum samples from putatively immune individuals has been applied (Bennuru et al., 2016). Recently a multi-epitope subunit vaccine coding for selected B-cell and T-cell epitopes, was constructed representing a novel approach for generating a specific immune response thereby avoiding responses against other unfavourable epitopes in the complete antigen (Shey et al., 2019). Another optional vaccine consists of nanoparticles or the use of non-protein molecules such 
as carbohydrates, like the specific glycoform of glycosyl-phosphatidylinositol, that can act as vaccine candidates, as indicated for microbes (Jaurique and Seeberger, 2017).

\subsection{Role of Wolbachia in onchocerciasis}

The Wolbachia endobacteria are obligatory symbionts contributing to the viability of the parasite, its growth and development. The endobacteria are transovarially transmitted like mitochondria to the next filarial generation. Thus, antibiotics deployed to antagonize the endobacterial symbiotic role result in disruption of embryogenesis in female filariae.

Immunologically, the Wolbachia stimulate innate and adaptive immune responses. The bacteriaderived surface-associated and released molecules play immunological and pathological roles in onchocerciasis. In particular, endotoxin-like molecules induce Th1-type inflammatory reactions as known in all gram-negative lipopolysaccharide-exposing bacteria (Brattig et al., 2000; 2004b). In contrast to the helminth-characteristic type 2 (Th2) and Th3 immune responses, the Wolbachia provoke bacteria-typical predominant type 1 (Th1) reactions. Neutrophils - characteristically activated against bacteria accumulate within an onchocercoma at the surface of a female comprising a multitude of Wolbachia in the lateral cord (see section 3.3., Fig. 7.5) (Brattig et al., 2001; Tamarozzi et al., 2016) - but neutrophils are absent in the onchocercoma on the surface of a female when the onchocerciasis patient has been treated with antibacterial doxycycline eliminating the Wolbachia (Fig. 9) (Brattig et al., 2001; 2004; Pearlman, 2003; Saint André et al., 2002). Subsequently, high peripheral levels of TNF-alpha, IL-1 beta, IL-6, IL-8 and antibacterial acute phase reactants arise on site and in the circulation. The Wolbachia surface protein (WSP) and heat-shock protein induce Th1-associated cytokines, TLR2/4 and IgG1 antibody responses (Pearlman, 2003; Brattig, 2004b; Kamalakannan et al., 2012; Tamarozzi et al., 2016).

The endotoxin-like and other products of the Wolbachia initiate a major proinflammatory stimulus in the eye disease leading to keratitis. Wolbachia, in addition, are associated with the severity of adverse reactions after chemotherapy of onchocerciasis with anti-filarial drugs. Wolbachia thus represent a target for therapy (Saint Andre et al., 2002; Pearlman, 2003).

\section{Onchocerciasis control and elimination programmes}

\subsection{Elimination of onchocerciasis by chemotherapy of infected patients}

Table 3 summarises past, present and potential future therapeutic agents for treatment of onchocerciasis. The first therapeutic agent against parasitic infections was suramin (Germanin; Bayer AG), a complex compound with four aromatic benzene rings and a functional urea group, which was introduced in 1949 (Wilson and Wormall, 1949). Suramin is a micro- and macrofilaricide, i.e. it not only kills microfilariae but also adult filariae. Suramin damages the intestinal epithelium of the filaria. Suramin, however, is inherently dangerous because of its high protein-binding affinity and alteration of enzyme 
function; thus, it carries the risk of dermatitis, diarrhoea, optic neuropathy, nephrotoxicity, and even the occasional death. A three-year study in the Onchocerciasis Control Programme advised against suramin treatment (Rolland et al., 1980) and it is contraindicated in pregnancy.

Diethylcarbamazine (DEC, Hetrazan. PharmaCompass) a piperazine derivative, has been used as therapy against onchocerciasis since 1950 (Ruiz Reyes, 1951). It is also a micro- and macrofilaricide affecting the neuromuscular system of the parasites and promotes cellular cytotoxicity mediated by immune factors. In addition, DEC provokes various side effects such as itching and urticaria (reactions to disintegrating microfilariae) facial swelling, headache, nausea, vomiting, fever, joint pain and anorexia. DEC is used in a patch test (Mazzotti) for detection of skin microfilariae (see section 3.2.).

Kuesel (2016) reviewed the path from discovery of new compounds (see below) to their qualification for large scale use and the support of regulatory authorities provides for development of drugs for neglected tropical diseases.

Ivermectin (Mectizan ${ }^{\mathrm{R}}$, Merck) a macrocyclic lactone, derived from Streptomyces, is an endectocide. In 2015, the Nobel Prize in physiology or medicine was jointly awarded to W. Campbell (University of Wisconsin) and S. Omura (Kitasato University) at the Karolinska Institute (Sweden) for their discovery and exploration of ivermectin and its mode of action, resulting in a novel therapy against onchocerciasis and other nematode infections (Aziz et al., 1982; Campbell et al., 1983, 2016; Ömura, 2016; van Voorhis et al., 2015) (Fig. 8). This molecule binds to the inhibitory neurotransmitter GABA on neurons and muscles resulting in an irreversible activation of a chloride influx, in a hyperpolarization of the membrane, and in paralysis and death of microfilariae. Ivermectin expresses micro- but not macrofilaricidal activity, although it causes long-term sterility of the adult female worms. Ivermectin was introduced for antifilarial treatment in 1981. Albiez et al. (1988b) showed that ivermectin was a more effective microfilaricidal agent than DEC that caused more frequent and severe side effects. Initially, ivermectin was

531 administered once a year $(150 \mu \mathrm{g} / \mathrm{kg}$ ), but lately it is increasingly administered bi-annually (Frempong et 532 al., 2016). A 3-monthly treatment with ivermectin even may be most effective to prevent the appearance 533 of onchocercomata (Campillo et al., 2020). The 3-monthly treatments not only target microfilariae, but 534 probably in addition the moulting of third to fourth stage larvae and possibly can affect immature adults suggesting a prophylactic effect. Further, Navarro et al. (2020) reviewed data on the safety of high doses of ivermectin ( $>400$ up to $800 \mu \mathrm{g} / \mathrm{kg}$ ) but did not exclude ocular adverse events.

Ivermectin is donated free of charge by the Mectizan Donation Program and was distributed amongst communities by the African Programme for Onchocerciasis Control (APOC) and by various Non-

539 Governmental Organisations (NGOs) such as Sight Savers, Lions International Sight First Programme, The

540 Carter Foundation and the Helen Keller Foundation. Latterly, the Expanded Special Project for

541 Elimination of Neglected Tropical Diseases (ESPEN) has responsibility for oversight of ivermectin

542 distribution in Africa. The programme reaches more than 300 million people in the affected areas of 35

543 countries annually, with more than 3.4 billion treatments donated since 1987. (The Mectizan ${ }^{\mathrm{R}}$ Donotion 
Program (MDP) https://www.merck.com/about/featured-stories/mectizan.html, https://www.cartercenter.org/health/river_blindness/index.html).

The drug has rare adverse effects such as red eyes and dry and burning skin. However, ivermectin is contraindicated in persons with loiasis due to the risk of ivermectin-associated severe inflammation since treatment with ivermectin may result in adverse reactions in patients with both onchocerciasis and loiasis

549 (Gardon et al., 1997) (see section 3.1.). In a 'test-and-treat' (TNT) strategy a rapid test (LoaScope) has

550 been introduced for loiasis-endemic areas identifying individuals with levels of Loa loa microfilaremia

551 associated with a risk of post-ivermectin severe adverse events. LoaScope-positive individuals were

552 excluded from ivermectin treatment (Boussinesq et al., 2018). Adverse effects after ivermectin treatment 553 have been observed at a rate of about 9\% with cases showing hypotension or dyspnoea (De Sole et al., 554 1989). Bockarie et al. (2013) discussed the option of preventive chemotherapy as a strategy for 555 elimination of onchocerciasis by treating populations at risk, to prevent transmission or morbidity.

Moxidectin, a milbemycin macrocyclic lactone, related to ivermectin, has been used since 1995 as an 557 anthelminthic in veterinary medicine against various Onchocerca species (Monahan et al., 1995). Opoku et 558 al. (2018) conducted a randomised, controlled, double-blind phase 3 trial in the Democratic Republic of 559 Congo, Ghana and Liberia and stated that skin microfilarial loads were lower after moxidectin treatment 560 than after ivermectin treatment. Moxidectin would therefore be expected to reduce parasite transmission between treatment rounds more than ivermectin could, thus accelerating progress towards elimination. Moxidectin has microfilaricidal and embryostatic effects after a single dose and expresses a macrofilaricidal effect upon repeated doses. Several studies indicate that moxidectin has a higher efficacy

564 than ivermectin (Awadzi et al., 2014). Besides moxidectin, also flubendazole and emodepside had been investigated as candidate drugs (Kuesel, 2016).

Recently, metabolic chokepoint compounds have been identified which were either produced or consumed by a single enzyme reaction. The respective checkpoint enzymes that govern these reactions

568 have been investigated (Taylor et al., 2013). Inhibition of such enzymes either leads to a toxic

569 accumulation or lack of a compound necessary for subsequent reaction. Taylor reported anti-filarial

570 effects on Onchocerca microfilariae by perhexiline, a piperidine derivative affecting carnitine o-

571 palmitoyltransferase and the fatty acid oxidation pathway. Most recently, benzimidazole-benzoxborole

572 hybrids, amide- or ketone-linked, termed 8a (AN8799) or 21 (AN15470), have been reported as promising

573 macrofilaricidal agents tested to date in animal models (Akama et al., 2020).

$574 \quad$ Since 1998 antibiotic therapy has demonstrated depletion of Wolbachia endobacteria in O. volvulus 575 and other filariae (Hoerauf et al., 2000, 2001). Doxycycline was proposed for treatment of onchocerciasis 576 in addition to ivermectin since adult females were sterilized when the antibiotic killed the Wolbachia (Fig. 577 9). However, a general implementation of doxycycline for filariasis therapy was hardly feasible because of 578 the frequency and duration of the required treatment of 100 or $200 \mathrm{mg}$ daily for 4-6 weeks. Also, adverse 579 reactions have been reported and no pregnant women and children can take doxycycline (Hoerauf et al., 
2003b, 2008; Abegunde et al., 2016).

Since 2014 an Anti-Wolbachia Consortium (A-WOL) at the Liverpool School of Tropical Medicine, has

582 been active among others in the field of antiwolbachial drug discovery to treat filarial infections. There are

583 numerous ongoing studies on novel alternate drugs against Wolbachia with excellent potential. The

584 tylosin analog ABBV-4083 (TylAMac), a macrolide antibiotic, is an inhibitor of bacterial protein synthesis.

585 ABBV-4083 resulted in a >99\% elimination of Wolbachia as measured 16 weeks after treatment initiation,

586 blocking the embryogenesis and leading to a complete clearance of circulating microfilariae. ABBV-4083

587 expressed relatively low activity against microfilariae of $L$. loa. A successfully completed phase I clinical

588 trial assessing the safety and tolerability of ABBV-4083 has provided encouraging findings to support

589 advancement of ABBV-4083 to phase II clinical trials (von Geldern et al., 2019;

590 https://www.dndi.org/diseases-projects/portfolio/abbv-4083/).

591 There are other attractive non-macrolid antibiotic anti-Wolbachial compounds (AWZ=anti-Wolbachia)

592 such as the heterocyclic thienopyrimidine/quinazoline scaffold AWZ1066 and its enantiomers AWZ1066-

593 S and -R expressing drug metabolism/pharmacokinetic features (Hong et al., 2019). AWZ1066S is a highly

594 specific anti-Wolbachia candidate selected through a lead optimization programme focused on balancing

595 efficacy, safety and drug metabolism/ pharmacokinetic (DMPK) features of a thienopyrimidine

596 /quinazoline scaffold derived from phenotypic screening. AWZ1066S shows superior efficacy to existing

597 anti-Wolbachia therapies in validated pre-clinical models of infection and has DMPK characteristics that

598 are compatible with a short therapeutic regimen of 7 days or less. This candidate molecule is well-

599 positioned for onward development and has the potential to make a significant impact on communities

600 affected by filariasis. Furthermore, some intriguing future anti-Wolbachial candidate molecules include

601 the heterocyclic quinazolines CRB417 and CRB490 with excellent efficacy and properties (Bakowski and

602 McNamara, 2019). Very recently, in vivo efficacy of boron-pleuromutilin AN11251 against Wolbachia in

603 the rodent filarial nematode Litomosoides sigmodontis model has been demonstrated to be superior to

604 doxycycline (Ehrens et al., 2020). Thus, AN11251 treatment resulted in a Wolbachia FtsZ/actin reduction

605 of $94 \%$ compared to $<40 \%$ with doxycycline.

606

607 


\begin{tabular}{|c|c|c|c|}
\hline Target & Therapeutic agents & Originator / Operator & $\begin{array}{c}\text { Start of } \\
\text { treatment }\end{array}$ \\
\hline Onchocerca & $\begin{array}{l}\text { Suramin } \\
\text { Diethylcarbamazine, DEC (Hetrazan) } \\
\text { Ivermectin } \\
\text { Moxidectin, Milbemycin }\end{array}$ & $\begin{array}{l}\text { Wilson and Wormall } \\
\text { Ruiz Reyes } \\
\text { Aziz } \\
\text { Campbell } \\
\text { Ömura } \\
\text { Nobel prize, Karolinska } \\
\text { Institute, Sweden } \\
\text { van Voorhis } \\
\text { Monahan } \\
\text { Opoku }\end{array}$ & $\begin{array}{l}1949 \\
1951 \\
1982 \\
1983,2016 \\
2016 \\
2015 \\
2015 \\
1995 \\
2018\end{array}$ \\
\hline Wolbachia & $\begin{array}{l}\text { Doxycycline } \\
\text { Doxycycline versus Ivermectin } \\
\text { Tolosin A analog: ABBV-4083, } \\
\text { TylAMac } \\
\text { AWZ1066S, CRB490/417 }\end{array}$ & $\begin{array}{l}\text { Hoerauf } \\
\text { Abegunde } \\
\text { von Geldern } \\
\text { Taylor } \\
\text { Hong }\end{array}$ & $\begin{array}{l}2002 \\
2016 \\
2019 \\
2019 \\
2019\end{array}$ \\
\hline
\end{tabular}

610

611

612

613

614

615

616

617

618

619

620

621

622

623

624

625

626

627

\subsection{Vector control}

\subsubsection{Vector control 1932-1974}

The first known attempt to control onchocerciasis by vector control was in Mexico in 1932. This and many other vector control efforts by both vegetation removal and chemical applications were reviewed by Davies (1994). There were aerial treatments with the organochlorine Dichloro-diphenyl-trichloro-ethane (DDT) of the River Congo at Kinshasa, now in the Democratic Republic of Congo, from 1948 to 1952 which led to the temporary disappearance of S. damnosum s.l., after which the vector populations have never recovered to their pre-control levels. DDT was in addition successfully used in Kenya and Uganda. In 1943, bush-clearing led to the disappearance by 1947 of vectors from the small $\left(42 \mathrm{~km}^{2}\right)$ Riana focus in Kenya and, in 1946, the vector $S$. neavei was eliminated from a focus in the Kodera district of Kenya by dripping DDT into rivers (McMahon et al., 1958), even though it was not known until 1950 that the species' immature stages were phoretic on crabs. Also, in Kenya, DDT was successfully used to eradicate onchocerciasis vectors from foci in Kissy/Kericho and North Nyaza. A similar success was achieved in neighbouring Uganda, when DDT was used again to eliminate S. damnosum s.l. from the Victoria Falls by 1973. 
Other control programmes were maintained in West Africa, for instance in Côte d'Ivoire from 1965 to 1971. DDT was phased out in favour of temephos, which was first used for Simulium control in the Sanaga river, Cameroon, in 1972, and temephos was the insecticide of choice used by OCP from 1975 (see below). This was after it was realised that localised control was only effective in isolated foci, when a plan for the massive Onchocerciasis Control Programme in the Volta Basin of West Africa (OCP) was initiated (https://www.who.int/blindness/partnerships/onchocerciasis_OCP/en/).

634

4.2.2. The World Health Organization Onchocerciasis Control Programme in the Volta Basin of West Africa (OCP)

At a meeting in Tunis during 1-8 July 1968 on the feasibility of onchocerciasis control it was agreed to plan a control campaign covering seven countries around the Volta Basin of West Africa (WHO/ONCHO/69.75 Joint US-AID/OCCGE/WHO Technical Meeting on the Feasibility of onchocerciasis control. Tunis, 1-8 July 1968, Report). This led to the production of a proposal to the Governments of Dahomey (now the Republic of Benin), Ghana, Ivory Coast (= Côte d'Ivoire), Mali, Niger, Togo and Upper Volta (now Burkina Faso) for the initiation of a control programme using aerial applications of insecticide to the vector's breeding sites in rivers (WHO, 1973)

The OCP programme was established in 1974 and spraying started in 1975, with the aim of interrupting transmission for twenty years to allow for all existing adult worms to die (WHO/OCP/1973). This would intend to protect areas previously abandoned due to the severity of the disease and allow re-population and increased agricultural production. It was thought at the time that 5.9 million people would have been infected in the above seven countries in 1975. Later the programme was extended to include Guinea, Guinea-Bissau, the western part of Mali, Senegal and Sierra Leone where a further 6.8 million people would have been infected, but revised estimates suggest that these were underestimates and that 17.8 million were infected in the 11 countries of the extended OCP (O'Hanlon et al., 2016).

Detailed descriptions of the history and the structure of the OCP, the methods, and the results during the first five years of the programme were provided by Walsh et al. $(1978,1979)$ and updated by Philippon et al. (1990). To assess the results of the vector control measures from November 1974 to October 1978 almost 1.2 million S. damnosum females were caught in over 52,000 man-days of catching and 674,000 flies were dissected to determine Annual Biting Rates (ABRs) and Annual Transmission Potentials (ATPs) (Walsh et al., 1978).

Up to $6000 \mathrm{~km}$ of rivers were sprayed weekly from the air in the original 7 countries in the dry seasons and 18,000 km in the rainy seasons. Eventually, vector control was expanded into the southern and western extension areas bringing the total OCP area to $1235000 \mathrm{~km}^{2}$ with a population of 30 million and increasing the lengths of rivers under control to $50000 \mathrm{~km}$ (Samba, 1994). 
pupae and it was deduced, and later shown by experimental treatments of potential sources, that there was a reinvasion of the treated zone by flies bred outside it (Garms et al., 1979b). It was later established that the flies involved in studies in Côte d'Ivoire, in the central OCP area, were mostly savannah members of the S. damnosum complex (S. damnosum s.str. and S. sirbanum) and that they could migrate enormous distances of up to $500 \mathrm{~km}$ (Baker et al., 1990). Furthermore, they were parous and many carried infective larvae so they were of epidemiological importance (Garms et al., 1979b). A similar phenomenon also occurred in the east of the OCP, where S. squamosum was additionally involved (Cheke and Garms, 1983), and in the west where savannah flies were found to migrate both northeastwards and southeastwards out of and into Guinea, respectively (Baker et al., 1990).

Despite supplementing insecticidal control with mass drug administration of the microfilaricidal compound ivermectin, onchocerciasis control was not complete in some areas. Some rivers continued to have S. damnosum s.l. larvae present after extensive treatment cycles and infective adult flies were still being caught. After the main OCP ceased operations in 2002 such areas, designated as special intervention zones, continued to be treated with insecticides until 2001).

The great success of the OCP by 2002 was to have freed for agriculture $250,000 \mathrm{~km}^{2}$ of fertile land from the threat of onchocerciasis, 40 million people had been protected from the disease and 600,000 cases of blindness prevented in seven countries (WHO, 2002). After the cessation of OCP, responsibility for continuing onchocerciasis control was devolved to the eleven member countries' governments.

\subsubsection{The African Programme for Onchocerciasis Control (APOC)}

In 1995 WHO had instigated the African Programme for Onchocerciasis Control (APOC) aiming to promote control (and from 2009 elimination) by establishing self-sustaining community-directed treatment with ivermectin (CDTI), and, where appropriate, vector control with environmentally safe methods. The participating 19 countries were the remaining non-OCP endemic countries: Angola, Burundi,

688 Cameroon, Central African Republic, Chad, Democratic Republic of Congo, Equatorial Guinea, Ethiopia,

689 Gabon, Kenya, Liberia, Malawi, Mozambique, Nigeria, Rwanda, Sudan, Tanzania and Uganda. APOC's vector 690 control activities were restricted to a few isolated foci, notably in Bioko (Equatorial Guinea) (Traoré et al., 691 2009), Tanzania and Uganda (Garms et al., 2009; https://www.who.int/apoc/vector/en/). APOC

692 terminated in December 2015 and WHO's action on onchocerciasis control was subsumed in May 2016

693 within the Expanded Special Project for Elimination of Neglected Tropical Diseases (ESPEN) that deals not

694 only with onchocerciasis but also with lymphatic filariasis, loiasis, schistosomiasis, soil-transmitted

695 helminthiasis and trachoma (http://espen.afro.who.int/; Hopkins, 2016). 
African Republic, Chad, Congo, Côte d'Ivoire, Democratic Republic of the Congo, Equatorial Guinea, Ethiopia, Ghana, Guinea, Guinea Bissau, Liberia, Malawi, Mali, Mozambique, Nigeria, Senegal, Sierra Leone,

702 South Sudan, Togo, Uganda and the United Republic of Tanzania. In the Eastern Mediterranean Region,

703 MDA continued in Sudan and Yemen. The results of evaluations of CDTI were separated into those

704 campaigns that were reported as being where criteria for stopping CDTI had been met or were close to 705 elimination, on track to elimination or showing unsatisfactory progress.

\subsubsection{Africa}

\subsubsection{Guinea}

The first research on onchocerciasis was conducted by staff of the Bernhard Nocht Institute in the 1960s. Knüttgen (1964) and Knüttgen and Büttner (1968) examined 18,634 people in northeastern

711 Guinea and reported that $45.8 \%$ carried microfilariae and $24 \%$ had nodules, with blindness rates varying 712 from 1.07 to 4.56\%. Subsequently, Knüttgen (1971) and De Sole et al. (1991) summarized the epidemiological situation. Garms and Post (1966) were the first to report the presence of S. damnosum s.l. and later Garms and Vajime (1975) found that, of the then known cytospecies, S. yahense, S. sanctipauli, $S$. soubrense, S. damnosum s.str. and S. sirbanum were present. This information was updated by Boakye et al. (1998). Guinea was part of the western extension of the OCP and was identified as a source of flies reinvading northern Cote d'Ivoire and Burkina Faso, involving distances of up to $500 \mathrm{~km}$ (Baker et al., 1990; see section 4.2.2.). According to WHO 2018a,b in 2017 almost 7 million people in 24 districts still required MDA with a reported coverage of $72.3 \%$.

\subsubsection{Liberia}

Liberia was never a member of the OCP but onchocerciasis and its vectors were studied extensively by teams from the Bernhard Nocht Institute for Tropical Medicine, which established the Liberian Research Unit field station at Bong town, in 1968. The extent of onchocerciasis in the country was described by Frentzel-Beyme (1973, 1975a,b). Interestingly, the clinical state of onchocerciasis known as sowda is frequent in Liberia (Darge and Büttner, 1995) (see section 3.3.). Garms and Vajime (1975a) showed that $S$. damnosum s.str. and $S$. sirbanum occurred in the savannah areas, while in the forest $S$. yahense and a variety of members of the S. sanctipauli sub-complex are present, of which S. yahense is the most important vector (Garms, 1987; Trpis, 2006). The form of the S. sanctipauli complex occurring in the St. Paul river was originally described by Vajime and Dunbar (1975) as S. sanctipauli s.str. but after reexaminations of cytotaxonomic material Post (1986) pointed out that its chromosome patterns actually showed it to be a form of $S$. soubrense.

Furthermore, those populations are genetically distinct from the Farmington form present in the

734 Farmington river (Kashan and Garms, 1987; Güzelhan and Garms, 1991). In addition S. sanctipauli s.str. 
rivers. Also, S. soubrense B (= S. leonense) was recorded in the Farmington river (Güzelhan and Garms, 1991). Anthropogenic factors have influenced vector dissemination as extensive deforestation associated with iron mining activities allowed savannah forms (S. damnosum s.str. and S. sirbanum) to invade previously forested areas as far south as Bong (Garms 1987; Garms et al., 1991). Treatments with ivermectin began in 1999 but Liberia has also been the site of successful trials of a new macro- and microfilaricide, moxidectin (Opoku et al., 2018; see section 4.1).

\subsubsection{Cameroon}

At the beginning of the 1990s it was estimated that 1,300,000 people were infected in Cameroon, of whom 26,000 were blind (WHO, 1995). Major advances in onchocerciasis research were made from a base at Kumba in Cameroon by Duke and his colleagues who developed the concept of Onchocerca-Simulium complexes involving forest and savannah strains of the parasite (Duke et al., 1966; Duke 1967a; Lewis and Duke, 1966).

Non-volvulus species of Onchocerca are more commonly found in S. damnosum s.l. in Cameroon than is usual in many other African countries (Duke 1967b). This necessitates care in the analysis of the results of Onchocerca parasites counted in the vectors. The most important of these is 0 . ochengi, a cattle parasite, the impact of which on the transmission of $O$. volvulus was discussed by Eisenbarth et al. (2016)

Detailed studies in Cameroon of the vectorial abilities of S. damnosum s.str. and S. sirbanum have been conducted (Renz 1987, Renz and Wenk 1987) and on S. squamosum B in the Sanaga valley by Demanou et al. (2003), who also discussed data from Kumba on S. squamosum A and C.

Studies in Cameroon have highlighted the slow progress towards elimination based on ivermectin. Katabarwa et al. (2013) described how only 3 of 11 health districts were close to elimination after 15 years of treatment. Continuing transmission and prevalence of up to $52.7 \%$ were also reported for areas in the southwest by Wanji et al. (2015a) after 10 years of ivermectin distribution by CDTI. In another study it was reported that $15.5 \%$ of 2,364 people had never taken ivermectin (Wanji et al., 2015b). Similar results with onchocerciasis remaining at mesoendemic levels in the Centre and Littoral Regions and in the Vina du Nord River Valley after 15 and 25 years of CDTI, respectively, were documented by Kamga et al.

763 (2016) and Eisenbarth et al. (2016).

It is unlikely that a long-term solution to the control of biting fly numbers or of onchocerciasis will be possible in the long-term without complementary vector control. Whilst, in theory, ivermectin distribution will interrupt transmission if distribution is maintained at $100 \%$ coverage for more than 25 years, in practice this is unlikely to be achieved because of (a) insufficient coverage for logistic and management reasons; (b) lack of acceptability of the drug in loiasis areas; and (c) the emergence of resistance to ivermectin, as "non-responders" (defined as individuals with microfilaria (mf) counts in skin

$770>10 \mathrm{mf} / \mathrm{snip}$ after nine or more rounds of ivermectin treatment) have already been found in Ghana

771 (Dadzie et al. 2003, Awadzi et al. 2004), with further evidence for it in Cameroon (Bourginat et al 2007). 

and $71 \%$ were treated.

\subsubsection{Uganda}

In Uganda, where approximately 1.4 million people had been infected with onchocerciasis (Ndyomugyenyi 1998) and the disease existed in 17 foci, about 2.8 million people required MDA in 2017 (WHO 2018a,b), but not in 8 formerly endemic districts after onchocerciasis had been eliminated by MDA and vector control. So far Uganda, together with Kenya, Equatorial Guinea, Sudan and Ethiopia are the only African countries where onchocerciasis foci have been eliminated. In Uganda onchocerciasis is transmitted by two vectors, both of which were described from there: S. damnosum Theobald 1903 and $S$. neavei Roubaud 1915 (Adler, 2019). The larvae of S. neavei develop in a phoretic association on freshwater crabs of the genus Potamonautes and S. neavei is or was the vector in most of the smaller isolated foci. Uganda was one of the first countries where large scale vector control projects were carried out. From 1951 to 1973 there were 11 vector control projects, all with DDT (Davies 1994). Particularly famous were the projects on the important Victoria Nile focus where $S$. damnosum was breeding in a series of $70 \mathrm{~km}$ of rapids below the Owen dam. The application of 1973 was completely successful (McCrae, 1978), no flies have been found up to the present day (Davies, 1994), but it had never been formally verified that the transmission had been stopped. However, a recent study showed a total of 2953 serum samples taken from children younger than ten years and tested using the Ov16 ELISA test (see section 3.2.) were all negative (Katabarwa et al., 2020). Fly catches were carried out at the historical catching sites for at least a year. No S. damnosum were collected, indicating that the former vector never came back. However, 854 Simulium adersi Pomeroy (Subgenus Meilloniellum) were caught, which all turned out to be negative when tested by PCR. S. adersi is not known to be a vector of onchocerciasis, but can be infected experimentally (Wegesa, 1970).

Of the original 17 onchocerciasis foci in Uganda (Fig. 10) in only one, the Lhubiriha focus in Kasese District bordering D.R. Congo (Fig. 10.2, focus 14; red) is transmission by S. kilibanum (S. damnosum complex) still continuing. Interruption of the transmission by S. damnosum has now probably been achieved in the Mid North Focus (Fig. 10.2, focus 14; light green), mainly by vector control using temephos,

800 but also experimentally by clearing of vegetation, window traps and CDTI. Vector control was also

801 primarily responsible for the elimination of the Kashoya-Kitomi S. neavei focus (Lakwo et al., 2017). In

802 most Ugandan foci where onchocerciasis has been eliminated by CDTI and vector control, Simulium neavei 803 was the vector. Many of such successes followed on from research and control work begun in 1991 in a 804 cooperation between the German Technical Cooperation Agency (GTZ), the Bernhard Nocht Institute for 805 Tropical Medicine, Basic Health Services Project, and the V,ector Control Unit of the Ministry of Health in 806 Kabarole District (now Kabarole and Kyenjojo districts) in Western Uganda. 
When annual distribution of ivermectin began in 1991 no vector control was planned, but treatments were accompanied by studies on the transmission by the vector $S$. neavei. After 4 years, there was no clear effect on the transmission and 1000 parous flies still had 151 infective larvae in their heads. In view of these results and at the request of the local government it was decided to enhance the effect by vector control. Before starting this, it had been confirmed that temephos, which had been used for 25 years in the

812 OCP (see section 4.2.2.), could be used safely, in particular, without harming the phoretic host crabs $P$. 813 Aloysiisabaudiae (Garms et al., 2017). After only a few monthly applications from mid-1995 to the end of 814 1996, S. neavei had disappeared from the main Itwara focus and never came back. Sub-foci on the Siisa 815 and Aswa rivers took a bit longer, but no positive crabs or biting flies were seen any more throughout the 816 focus after February 2003 (Garms et al., 2009; Michael et al., 2020).

Of especial interest was the Imaramagambo focus in south-western Uganda, where vector control had 818 been planned, but when it turned out that there was no transmission anymore, it was noticed that the 819 vector S. neavei and its phoretic host, the freshwater crab, had both disappeared, possibly because of 820 runoff into rivers of agricultural chemicals used intensively on the nearby tea plantations (Katabarwa et al., 2016). The flies also disappeared from areas in the Ruwenzori valley to the northwest of the Itwara focus (Garms et al., 1994) and habitat changes have also contributed to reductions in transmission elsewhere in Uganda, as Fischer et al. (1997) reported a reduced prevalence of onchocerciasis following deforestation. In addition, by 2017 MDA was leading to interruptions or suspected interruptions of transmission in five other foci where S. neavei was the vector (Katabarwa et al., 2018). The Madi Mid North focus, where $S$. damnosum s.l. is the vector, is likely to be the most intractable area but details of the cytoform present in that region have not been published. In western Uganda the following S. damnosum cytoforms have been recorded: S. kilibanum, "Sebwe", "Nkusi" and S. pandanophilum, of which only $S$. kilibanum is anthropophilic and of vectorial importance (Krüger et al., 1999).

Control of S. damnosum s.l. by removing the trailing vegetation upon which immature stages develop 832 partial success, and in Malawi in the early 1990s by destruction of the aquatic plant Hydrostachys sp. (M. J. 833 Roberts, unpubl., Burnham 1992, Davis, 1994). Also, Baker and Abdelnur (1986a,b) showed that in a rocky 834 breeding site of the Bussere River in south-western Sudan a small team armed with axes, saws and sickles 835 could do much to reduce larval and pupal supports in the breeding sites caused by vegetation trailing in 836 fast water flow. A similar strategy has also recently been applied in Uganda (Jacob et al., 2018; Smith et al. 837 2019) but it is unlikely to provide a long-term solution as it requires regular "slash and clear" and, besides, 838 the vectors will adapt and breed on other substrates such as rocks, which they often use when no trailing 839 vegetation is present. In addition, large torrential rivers and rapids, particularly those in the middles of 840 wide rivers cannot be completely cleared. 
As mentioned in the historical introduction, Ghana was the site where onchocerciasis was first recorded (O'Neill, 1875). Crisp (1956) described the geographical extent and severity of the disease in the north and provided plans for a vector control campaign, while Waddy (1969) elaborated these with proposals that culminated in the OCP. Ghana was one of the original seven OCP countries, with the west of the country targeted at the outset in 1975. Most of the rest of the country north of the Volta Lake was included in Phase II soon afterwards, with areas south of the lake included from 1988 onwards as part of the southeastern extension. Most of the southwestern forested areas were not included in the vector control campaign, but were subject to MDA with ivermectin, following the first successful trials that were conducted in 1987 at Asubende on the River Pru (Remme et al., 1989).

The initial euphoria about possible elimination of onchocerciasis from Africa using ivermectin distributions was dealt a blow when incipient resistance to the drug was detected in Ghanaian patients (Awadzi et al., 2004). Later, cohorts of patients were found in the Brong-Ahafo and Northern Regions to be being re-populated with microfilariae sooner than was to be expected and these "non-responders" (OseiAtweneboana et al., 2007) were also possibly harbouring resistant worms (Osei-Atweneboana et al., 2011).

Despite the activities of the OCP and subsequent continuations of ivermectin distributions, some transmission continues (Kutin et al., 2004, Garms et al., 2015), including in areas where there had been vector control (Lamberton et al., 2015, F.B.D. Vereigh, pers.comm.to RAC July 2019).

The ivermectin distributions were originally annual but it is now the policy in Ghana to distribute biannually. This has succeeded in reducing transmission and infection rates in some areas but not everywhere that has been studied (Frempong et al., 2016, F.B.D. Vereigh, pers. comm.). Another consideration relevant to continuing transmission is the lack of complete compliance with the drug distribution programmes. Agyemang et al. (2018) investigated compliance in the Upper Denkyira East

866 Municipal area and reported that it was lower than given in official reports, with results ranging from 7 to $86751 \%$ with an overall compliance of only $21 \%$, even less than the $24.4 \%$ reported earlier by Kutin et al. 868 (2004).

869 Ghana has a high diversity of S. damnosum cytoforms, including some sites where up to five different 870 varieties could be found breeding sympatrically. Details of the vectors present and how their geographical 871 distributions have varied from the 1970s until 2011 were summarized by Post et al. (2013), with 872 fluctuations attributable to deforestation (Wilson et al., 2002), extinction due to vector control (Cheke et 873 al., 2008) and pollution resulting from illegal gold-mining activities in rivers such as the Pra and Offin 874 (Garms et al., 2015), although the mining (locally known as "Galamsy") has now been curtailed by 875 Government actions. According to WHO (2018a,b) in Ghana in 2017 of about 8 million infected people 4.4 876 million (54,6\%) in 84 of 85 endemic districts received MDA. 
Onchocerciasis is highly endemic in Ethiopia, with more than 20 million people infected or at risk

880 (Anon, 2015). The disease is mostly found in southwestern, western and northwestern parts of the

881 country, being particularly associated with coffee growing areas in the southwest and with cotton and oil

882 seed farming areas in the northwest. The main vectors are members of the $S$. damnosum complex (Hadis et

883 al. 2005), but S. ethiopiense, a member of the S. neavei group phoretic on crabs (Potamonautes antheus), is

884 suspected of being a secondary vector in the southwestern midlands and the highlands where it is often

885 sympatric with S. damnosum s.l. (White 1977).

886 Onchocerciasis control with ivermectin began in 2001 and by 2015 there were 18 CDTI project zones in 887 the country (Anon 2015). At present the country has some areas in various stages of post treatment 888 surveillance (PTS), for instance in the Metema area (see below; 4.3.1.7.). Onchocerciasis has disappeared 889 from the Tigray region in the absence of any control measures, probably in the wake of human migrations 890 and the establishment of commercial farming (Katabarwa et al., 2014b). In 2017, 17.5 million people 891 required MDA, with 194 of 199 endemic districts receiving it (WHO 2018a,b).

892

\subsection{Sudan}

There are three main areas in Sudan where onchocerciasis occurs or occurred. Principal amongst these was the Abu Hamed focus in River Nile State where the first case of the elimination of an onchocerciasis focus in Africa was achieved following ivermectin distribution (Zarroug et al., 2016), although it was probably assisted by the flooding of all of the western breeding sites of the vector in the River Nile by the construction of the Merowe dam (Zarroug et al., 2014). The vector there is a unique form, the hamedense form of $S$. damnosum (Higazi et al., 2001), which had led to prevalence of $37 \%$ in populations of up to 120,000 people, with high proportions of sowda (see section 3.3.) guidelines for interruption of transmission of $O$. volvulus, has been achieved at the cross-border focus

904 where the Galabat focus adjoins the Ethiopian Metema focus. This success, the first such cross-border initiative in Africa, was accomplished by a combination of annual and semi-annual ivermectin MDA

906 (Katabarwa et al. 2020b).

\subsection{South Sudan}

About half the population of South Sudan is affected by onchocerciasis with particularly high endemicity in Western Equatoria, and the Northern and Western Bahr el Ghazal areas. CDTI was begun in the mid-1990s and 5,605,726 people were being targeted in 2009 (Lugga and Chane 2011). Recent zones

912 for CDTI were mapped in the context of research on control of nodding syndrome in the country. 913 Pioneering investigations on the biology of the vectors was conducted in South Sudan by Lewis (1953) 914 and by Baker and Abdelnur (1986). 
Yemen is the only country in the Eastern Mediterranean with onchocerciasis, where it is most prevalent along the permanent waterways (wadis) draining into the Red Sea in the west of the country.

919 The vector breeds in very shallow flat wadis with low discharges of up to $1 \mathrm{~m}^{3} \cdot \mathrm{sec}^{-1}$ (Garms and Kerner, 920 1982). It is a unique species S. rasyani, a member of the S. damnosum complex (Garms et al., 1988).

921 Although planned, to our knowledge, there has been no vector control but sporadic ivermectin 922 distribution was begun in the early 1990s. Since 2011 civil strife and wars have interrupted the national 923 campaign. The disease in Yemen is characterised by high rates of the hyperreactive clinical manifestation 924 known as sowda (Anderson et al., 1973; Büttner et al., 1982; Büttner and Racz, 1983). Mahdy et al. (2018) 925 reported an overall seroprevalence rate of 18.5\% during their surveys in 2017. According to WHO 926 (2018a,b) in Yemen in 2017 of about 6.3 million people in 33 districts were requiring MDA.

\subsubsection{Americas (OEPA)}

In the continent of America onchocerciasis was restricted to six countries of central and south America: Brazil, Colombia, Ecuador, Guatemala, Mexico and Venezuela (Fig. 4). Differing from Africa with its prevalence in large parts of 31 countries, in the Americas the occurrence of onchocerciasis was or is confined to limited foci. Accordingly, the combat against onchocerciasis was different and an elimination of the infection appears to have been or will be feasible by mass drug administration with ivermectin.

A variety of vectors is or was responsible for transmission in central and southern America. Members of the S. ochraceum and S. metallicum complexes were involved in Mexico, with the former most active in transmission in Guatemala (Garms, 1975b; Rodríguez-Pérez et al., 2015). A detailed review of the potential importance of further anthropophilic Simulium species as vectors of $O$. volvulus in Guatemala has been presented by Takaoka (2015). toward elimination of onchocerciasis in the Americas. From 1989 to 2016, more than 11 million ivermectin treatments, given twice or four times per year, have been given in the Americas, eliminating transmission in

94311 of 13 foci. The number of people at risk of onchocerciasis decreased from $>530$ thousand to about 30

944 thousand. Nodulectomy campaigns, e.g. in Mexico and Guatemala, probably helped the success of the MDA

945 (Figueroa Marroquin,1975).

946 Onchocerciasis was eliminated in Columbia in 2010, in Guatemala 2011, in Ecuador 2012, in Mexico

9472014 and in Venezuela in 2017 apart from a focus in the South. One focus also exists in the north of Brazil.

948 In the remaining Amazonas focus that straddles the Venezuela-Brazil border the main vectors are

949 members of the S. oyapockense and S. guianense species complexes and S. incrustatum (Shelley et al. 2010). 
The OEPA's success influenced programmes in Africa, especially in Sudan and Uganda, which moved from a control to an elimination strategy in 2006 and 2007, respectively. The successes in the Americas have also influenced WHO guidelines for onchocerciasis transmission elimination. With four of the six originally endemic American countries now having eliminated onchocerciasis transmission, and 95\% of ivermectin treatments in the region halted, the regional focus is now on the remaining active transmission zone on the border between Venezuela and Brazil.

956

\section{Future perspective}

Recent publications report and discuss the success of elimination or close to elimination of onchocerciasis in limited foci in Africa - in Sudan, Mali, Senegal, Burundi, Chad, Malawi and Nigeria (Tekle et al., 2012; Zarroug et al., 2014, 2016; Walker et al., 2017; Rebollo et al., 2018; Richards et al., 2020). This success is completely or mainly based on MDA, of ivermectin. Exceptions are Bioko, Equatorial Guinea, where vector control was successful, and Uganda where onchocerciasis was eliminated from several isolated foci by combinations of control of the vector $S$. neavei by ground larviciding with temephos and MDA (Katabarwa et al 2018, 2020a,b; Michael et al., 2020).

The priority is given to treatment with ivermectin. Recently, attention, however, is drawn to the problems of poor coverage and inadequate compliance to MDA (Agyemang et al., 2018; Dissak-Delon et al., 2019). Correspondingly, Verver et al. (2018) discussed why a wide-reaching elimination of onchocerciasis cannot be guaranteed by 2025 and proposed a long-term biannual or quarterly MDA combined with vector control activities as complementary approaches (Routledge et al., 2018) for high-endemicity areas to accelerate progress toward elimination. The proof-of-principle in distinct foci in Mali, Senegal and Sudan indicate a possibility to eliminate onchocerciasis with annual or 6-monthly ivermectin treatment in some endemic foci in Africa. Correspondingly, the published WHO 2030 goals for onchocerciasis were influenced by the cited models (EPIONCHO and ONCHOSIM) on the impact of biannual or quarterly ivermectin treatment frequency and in addition complementary vector control (NTD Modelling Consortium Onchocerciasis Group, 2019; Gates Open Research 2019; Hassan and Shaban, 2020).

Anthelminthic drug alternatives to ivermectin, include the aforementioned Moxidectin (Awadzi et al. 2014, Opoku et al., 2018) and novel anti-Wolbachia agents like the thienopyrimidine/quinazoline scaffold AWZ1066 (Hong et al., 2019).

The timelines of onchocerciasis from control to elimination and eradication were discussed by Kim et al. (2015). They estimated that the elimination scenario will endure until 2028 in all endemic countries except four (Republic of Congo, Central African Republic, South Sudan, Gabon) but CDTI was predicted to continue beyond 2045 in countries with operational challenges, with around 1.15 billion treatments. The

983 elimination of transmission (EOT) of onchocerciasis for the majority of foci in the 34 countries in Africa

984 has been projected in several publications to be between 2025 and 2045 (Dadzie et al., 2018;

985 Gebrezgabiher et al., 2019; Kim et al., 2015). 
One major problem is that an elimination of onchocerciasis with ivermectin treatment alone has not so far appeared to be feasible in many African countries where onchocerciasis was endemic over millions of square kilometres spanning more than 30 countries. In addition, the vectors are highly efficient and with much higher endemicity levels migrating over hundreds of kilometres (Dadzie et al., 2003) threatening re-emergence of infected vectors and of onchocerciasis in their wake. Thus, exemplarily, on Bioko where the unique endemic vector (the Bioko form of $S$. yahense) was rendered extinct (Traore et al 2009) some vectors may have returned. These are S. squamosum rather than S. yahense (D. Boakye, pers, comm 2019) and it is unknown if they brought any 0 . volvulus with them.

Nevertheless, given that after nearly 15 years without any transmission on the island very few onchocercal cases remained (Hernández-González et al., 2016; Moya et al., 2016; Herrador et al., 2018; Ta et al., 2018), prospects for confirming elimination are good. The interruption of transmission was considered to have been permanent, but the Bioko case is salutary and illustrates that, however good planning and forecasts can be, there is room for the unexpected to affect our perspectives. In contrast, in the Americas, onchocerciasis elimination with ivermectin treatment has been considered feasible, since most onchocerciasis foci in the Americas were small and circumscribed, and most vector species are relatively inefficient. Thus, interruption of the transmission was feasible by 6-monthly or even 3-monthly ivermectin treatments (Sauerbrey et al., 2018).

Planning MDA programmes is now often based on the outputs of mathematical models such as ONCHOSIM (Plaisier et al 1990) or EPIONCHO (Basáñez et al. 2016) but these models skimped on details of blackfly biology. Only recently have models begun to model vector biology explicitly and started to take account of future uncertainties regarding climate change (Cheke et al., 2015) and the likelihood of needing to supplement MDA with vector control (Routledge et al., 2018). Such vector control in isolated foci could include ground larviciding, slash-and-clear vegetation destruction and killing host-seeking adult female flies in traps such as the Esperanza window trap (Rodriguez-Pérez, 2013; Toé et al., 2014; Hendy et al., 2017; NTD Modelling Consortium Onchocerciasis Group, 2019; NTD Modelling Consortium, Gates Open Research, 2019). These could be supplemented by deploying traps to catch ovipositing female flies such as “Bellec plates” placed beside breeding sites (Bellec, 1976; Cheke et al., 1982).

Difficulties surrounding the elimination of the transmission of onchocerciasis were reviewed by Cheke (2017), who drew attention to cases of successful control where transmission had probably been eliminated in contrast to areas where such interruptions were likely to be only temporary. These included areas in Africa with $>55 \%$ prevalence, where mass drug administration (MDA) alone was thought of as unlikely to succeed.

In summary, anthelminthic MDA complemented by appropriate vector control measures may increasingly lead to control and hopefully eradication of onchocerciasis which may be fulfilled in midcentury. 
1024 this review.

Abegunde, A.T., Ahuja, R.M., Okafor, N.J. 2016. Doxycycline plus ivermectin versus ivermectin alone for treatment of patients with onchocerciasis. Cochrane Database Syst Rev. 1, CD011146. Review

Adjobimey, T., Hoerauf, A. 2010. Induction of immunoglobulin G4 in human filariasis: An indicator of immunoregulation. Induction of immunoglobulin G4 in human filariasis: an indicator of immunoregulation. Ann. Trop. Med. Parasitol. 104, 455-464.

Abraham, D., Leon, O., Schnyder-Candrian, et al., 2004. Immunoglobulin E and eosinophil-dependent protective immunity to larval Onchocerca volvulus in mice immunized with irradiated larvae. Infect. Immun. 72, 810-817.

Achukwi, M.D., Harnett, W., Enyong, P., et al., 2007. Successful vaccination against Onchocerca ochengi infestation in cattle using live Onchocerca volvulus infective larvae. Parasite Immunol. 29, 1131111316.

Adler, P. H., Cheke, R.A., Post, R.J. 2010.) Evolution, epidemiology, and population genetics of black flies (Diptera: Simuliidae). Infection, Genetics, Evolution 10: 846-865.

Adler, P.H. 2019. World blackflies (Diptera: Simuliidae): a comprehensive revision of the taxonomic and geographical inventory. https://biomia.sites.clemson.edu/pdfs/blackflyinventory.pdf

Agyemang, A.N.O., Badu, K., Baffour-Awuah, S., et al., 2018. Evaluation of onchocerciasis control in the Upper Denkyira East municipal in the forest area of Ghana: Responses of participants and distributors to the CDTI programme. Acta Trop. 185, 357-362.

Akama, T., Freund, Y.R., Berry, P.W., et al., 2020. Macrofilaricidal benzimidazole-benzoxaborole hybrids as an approach to the treatment of river blindness: part 1. amide linked analogs. ACS Infect. Dis. 6, 173179.

Albiez, E.J., Ganley, J.P., Büttner, D.W. 1981. Ocular onchocerciasis in a hyperendemic village in the rain forest of Liberia. Trop. Med. Parasitol. 32, 25-28.

Albiez, E.J., Büttner, D.W., Duke, B.O. 1988a. Diagnosis and examination of nodules in human onchocerciasis. Trop. Med. Parasitol. 39 Suppl 4, 331-346. Review.

Albiez, E.J., Newland, H.S., White, A.T., et al., 1988b. Chemotherapy of onchocerciasis with high doses of diethylcarbamazine or a single dose of ivermectin: microfilaria levels and side effects. Trop. Med. Parasitol. 39, 19-24.

Alhassan, A., Osei-Atweneboana, M.Y., Kyeremeh, K.F., et al., 2016. Comparison of a new visual isothermal nucleic acid amplification test with PCR and skin snip analysis for diagnosis of onchocerciasis in humans. Mol . Biochem. Parasitol. 210, 10-12.

Al-Kubati, A.S., Mackenzie, C.D., Boakye, D., et al., 2018. Onchocerciasis in Yemen: moving forward towards an elimination program. Int Health. 10, Suppl. 1, i89-i96.

Allen, J.E., Sutherland, T.E. 2014. Host protective roles of type 2 immunity: parasite killing and tissue repair, flip sides of the same coin. Semin. Immunol. 26, 329-340. Review.

Amazigo, U., 2008. The African Programme for Onchocerciasis Control (APOC). Ann. Trop. Med. Parasitol. 102, Suppl 1, 19-22.

Anderson, J., Fuglsang, H., Al-Zubaidy, A. 1973. Onchocerciasis in Yemen with special reference to sowda. Trans. Roy. Soc. trop. Med. Hyg. 67, 30-31.

Andrews, J.A., Bligh, W.J., Chiodini, P.L., et al., 2008. The role of a recombinant hybrid protein based Elisa for the serodiagnosis of Onchocerca volvulus. J. Clin. Pathol. 61, 347-351.

Anon. 2015. Guidelines for onchocerciasis elimination in Ethiopia. Ministry of Health of the Federal Democratic Republic of Ethiopia. Addis Ababa, Ethiopia. 90 pages. Available from https://www.cartercenter.org/resources/pdfs/news/health publications/river blindness/ Onchocerciasis-Elimination-Certification-Guidelines-Ethiopia.pdf.

Awadzi, K., Boakye, D.A., Edwards, G., et al., 2004. An Investigation of persistent microfilaridermias despite multiple treatments with ivermectin, in two onchocerciasis-endemic foci in Ghana. Ann. Trop. Med. Parasitol. 98, 231-249.

Awadzi, K, Opoku, N.O., Attah, S.K., et al., 2014. A randomized, single-ascending-dose, ivermectincontrolled, double-blind study of moxidectin in Onchocerca volvulus infection. PLoS Negl. Trop. Dis. 8, e2953. 
Awadzi, K., Opoku, N.O., Attah, S.K., et al. 2015. Diagnosis of $O$. volvulus infection via skin exposure to diethylcarbamazine: clinical evaluation of a transdermal delivery technology-based patch. Parasit. Vectors 8, 515.

Aziz. M.A., Diallo, S., Diop, I.M., et al., 1982. Efficacy and tolerance of ivermectin in human onchocerciasis. Lancet. 2, 171-173.

Bain, O., Philippon, B., Séchan, Y., et al., 1976. Correlation between the number of microfilaria ingested and the thickness of the peritrophic membrane of the vector in onchocercosis of the African savannah. C. R. Acad Hebd Seances Acad. Sci. D. 283, 391-392.

Bain, O. 2002. Evolutionary relationships among filarial nematodes. pp. 21-29 in Klei, T.R. and Rajan, T.V. (Eds). The Filaria. Boston, Dordrecht and London, Kluwer Academic Publishers

Baker, R.H.A., Abdelnur, O.M. 1986. Localised onchocerciasis vector control in the Bahr el Ghazal Region of South-Western Sudan. II. Control. Trop. Med. Parasitol. 37, 135-142.

Baker, R.H., Guillet, P., Sékétéli, A., et al., 1990. Progress in controlling the reinvasion of windborne vectors into the western area of the Onchocerciasis Control Programme in West Africa. Philos. Trans. R. Soc. Lond. B Biol. Sci. 328, 731-747.

Bakowski, M.A., McNamara, C.W. 2019. Advances in antiwolbachial drug discovery for treatment of parasitic filarial worm infections. Trop. Med. Infect. Dis. 4, 108.

Bandi, C., Slatko, B., O'Neill, S.L. 1999. Wolbachia genomes and the many faces of symbiosis. Parasitol. Today. 15, 428-429.

Bandi, C., Trees, A.J., Brattig, N.W. 2001. Wolbachia in filarial nematodes: evolutionary aspects and implications for the pathogenesis and treatment of filarial diseases. Vet. Parasitol.,98, 215-238. Review

Bartlett, A., Bidwell, D.E., Voller, A. 1975. Preliminary studies on the application of enzyme immunoassay in the detection of antibodies in onchocerciasis. Tropenmed. Parasitol. 26, 370-374.

Bartlett, A., Turk, J., Ngu, J., et al., 1978. Variation in delayed hypersensitivity in onchocerciasis. Trans. R. Soc. Trop. Med. Hyg. 72, 372-377.

Basáñez, M.G., Walker, M., Turner, H.C., et al., 2016. River blindness: mathematical models for control and elimination. Adv. Parasitol. 94:247-341.

Bellec, C. 1976. Captures d'adultes des Simulium damnosum Theobald, 1903 (Diptera: Simuliidae) à l'aide de plaques d'aluminium, en Afrique de l'Ouest. Cahiers O.R.S.T.O.M. Série Entomologie Médicale et Parasitoligie 14, 209-217.

Bennuru, S., Cotton, J.A., Ribeiro, J.M., et al., 2016. Stage-specific transcriptome and proteome analyses of the filarial parasite Onchocerca volvulus and its Wolbachia endosymbiont. mBio 7, e02028- e02116.

Bennuru, S, O'Connell, E.M., Drame, P.M., et al., 2018. Mining filarial genomes for diagnostic and therapeutic targets. Trends Parasitol. 34, 80-90.

Bennuru, S., Oduro-Boateng, G., Osigwe, C., et al., 2020. Integrating multiple biomarkers to increase sensitivity for the detection of Onchocerca volvulus infection. J. Infect. Dis . 221, 1805-1815.

Blacklock, D.B., 1927. The insect transmission of Onchocerca volvulus (Leuckart, 1893). The cause of worm nodules in man in Africa. Br. Med. J. 1; 3446, 129-133.

Boakye, D., Back, C., Fiasorgbor, G.K., et al., 1998. Sibling species distributions of the Simulium damnosum complex in the west African Onchocerciasis Control Programme area during the decade 1984-93, following intensive larviciding since 1974. Med. Vet. Entomol . 12, 345-58.

Bockarie, M.J., Kelly-Hope, L.A., Rebollo, M., et al., 2013. Preventive chemotherapy as a strategy for elimination of neglected tropical parasitic diseases: endgame challenges. Philos. Trans. R. Soc. Lond. B Biol, Sci. 368, 20120144.

Bourguinat, C., Pion, S.D., Kamgno, J., et al., 2007. Genetic selection of low fertile Onchocerca volvulus by ivermectin treatment. Plos Negl. Trop. Dis. 1:e72

Boussinesq, M., Fobi, G., Kuesel, A.C. 2018. Alternative treatment strategies to accelerate the elimination of onchocerciasis. Int. Health. 10(suppl_1), i40-i48.

Bradley, J.E., Helm, R., Lahaise, M., et al., 1991. cDNA clones of Onchocerca volvulus low molecular weight antigens provide immunologically specific diagnostic probes. Mol. Biochem. Parasitol. 46, 219-227.

Bradley, J.E., Unnasch, T.R. 1996. Molecular approaches to the diagnosis of onchocerciasis. Adv. Parasitol. 37, 57-106. Review.

Brattig, N.W., Tischendorf, F.W., Albiez, E.J., et al., 1987. Distribution pattern of peripheral lymphocyte subsets in localized and generalized form of onchocerciasis. Clin. Immunol. Immunopathol. 44, 149159.

Brattig, N.W., Tischendorf, F.W., Strote, G., et al., 1991. Eosinophil-larval-interaction in onchocerciasis: heterogeneity of in vitro adherence of eosinophils to infective third and fourth stage larvae and microfilariae of Onchocerca volvulus. Parasite Immunol. 13, 13-22. 
Brattig, N.W., Krawietz, I., Abakar, A.Z., et al., 1994. Strong IgGisotypic antibody response in sowdah type onchocerciasis. J. Infect. Dis. 170, 955-961.

Brattig, N., Nietz, C., Hounkpatin, S., et al., 1997. Differences in cytokine responses to Onchocerca volvulus extract and recombinant Ov33 and OvL3-1 proteins in exposed subjects with various parasitologic and clinical states. J. Infect. Dis. 176, 838-842.

Brattig, N.W., Rathjens, U., Ernst, M., et al., 2000. Lipopolysaccharide-like molecules derived from Wolbachia endobacteria of the filaria Onchocerca volvulus are candidate mediators in the sequence of inflammatory and antiinflammatory responses of human monocytes. Microbes Infect. 2, 1147-1157.

Brattig, N.W., Büttner, D.W., Hoerauf, A. 2001. Neutrophil accumulation around Onchocerca worms and chemotaxis of neutrophils are dependent on Wolbachia endobacteria. Microbes Infect. 3, 439-446.

Brattig, N.W., Lepping, B., Timmann, C., et al., 2002. Onchocerca volvulus-exposed persons fail to produce interferon-gamma in response to 0 . volvulus antigen but mount proliferative responses with interleukin-5 and IL-13 production that decrease with increasing microfilarial density. J. Infect. Dis. 185, 1148-1154.

Brattig, N.W. 2004a. Pathogenesis and host responses in human onchocerciasis: impact of Onchocerca filariae and Wolbachia endobacteria. Microbes Infect. 6, 113-128. Review.

Brattig, N.W., Bazzocchi, C., Kirschning, C.J., et al., 2004b. The major surface protein of Wolbachia endosymbionts in filarial nematodes elicits immune responses through TLR2 and TLR4. J. Immunol. $173,437-445$.

Brumpt, E., 1919. Une nouvelle filaire pathogène parasite de l'homme isẹp: (Onchocerca caecutiens, n. sp.). Bull. $\quad$ Soc. Pathol. Exot. Filiales 12, 464-473 sispe?

Burbelo, P.D., Leahy, H.P., Iadarola, M.J., Nutman, T.B. 2009. A four-antigen mixture for rapid assessment of Onchocerca volvulus infection. PLoS Negl Trop Dis. 3, e438.

Burchard, G.D., Büttner, D.W., Bierther, M. 1979. Electron microscopical studies on onchocerciasis. III. The onchocerca-nodule. Tropenmed. Parasitol. 30, 103-112.

Büttner DW, von Laer G, Mannweiler E., et al., 1982. Clinical, parasitological and serological studies on onchocerciasis in the Yemen Arab Republic. Tropenmed Parasitol. 33, 201-212.

Büttner DW, Rácz P. 1983. Macro- and microfilariae in nodules from onchocerciasis patients in the Yemen Arab Republic. Tropenmed Parasitol. 34, 113-121.

Büttner, D.W. 1984. Onchocerciasis. Internist (Berl). 25, 229-235. Review

Caballero, C. E.., Barrera, A. 1958. Estudios helmintológicos de la region onchocercosa de Mexico y de la Repùblica de Guatemala. Nematodeda Iia Parte. Filaroidea V. Hallozgo de un nodulo oncocerciso en un mono arana Ateles geoffroyi vellerosus Gray, del Estado de Chiapas. Re'v. Lat. Amer. Microbiol. 7, $79-94$

Calamari, D., Yameogo, L., Hougard, J.M., et al., 1998. Environmental assessment of larvicide use in the Onchocerciasis Control Programme. Parasitology Today 14, 485-489.

Campbell, W.C., Fisher, M.H., Stapley, E.O., et al., 1983. Ivermectin: a potent new antiparasitic agent. Science. 221, 823-828.

Campbell, W.C. 2016. Ivermectin: A Reflection on Simplicity (Nobel Lecture). Angew Chem Int Ed Engl. 55, 10184-10189.

Campillo, J.T., Chesnais, C.B., Pion, S.D.S., et al., 2020. Individuals living in an onchocerciasis focus and treated three-monthly with ivermectin develop fewer new onchocercal nodules than individuals treated annually. Parasit. Vectors. 13, 258.

Cantey, P.T., Roy, S.L., Boakye, D., et al., 2018. Transitioning from river blindness control to elimination: steps toward stopping treatment. Int. Health. 10 (suppl 1); i7-i13.

Centers for Disease Control and Prevention (CDC).2013. Progress toward elimination of onchocerciasis in the Americas - 1993-201. MMWR Morb Mortal Wkly Rep. 62, 405-408.

Cheke, R. A., Garms, R., Kerner, M. 1982. The fecundity of Simulium damnosum s.l. in northern Togo and Infections with Onchocerca spp. Ann. Trop. Med. Parasit. 76: 561-568.

Cheke, R.A., Garms, R. 1983. Reinfestations of the southeastern flank of the Onchocerciasis Control Programme area by windborne vectors. Phil. Trans. R. Soc. Lond. B 302, 471-484.

Cheke, R.A., Fiasorgbor, G.K., et al., 2008. Elimination of the Djodji form of the blackfly Simulium sanctipauli sensu stricto as a result of larviciding by the WHO Onchocerciasis Control Programme in West Africa. Med. Vet. Entomol. 22, 172-174.

Cheke, R.A., Garms, R. 2013. Indices of onchocerciasis transmission by different members of the Simulium damnosum complex conflict with the paradigm of forest and savanna parasite strains. Acta Trop. 125, 43-52.

Cheke, R.A., Basáñez, M.G., Perry, M., et al., 2015. Potential effects of warmer worms and vectors on onchocerciasis transmission in West Africa. Philos. Trans. R. Soc. Lond. B Biol .Sci. 370, 1665. 
Cheke, R.A. 2017. Factors affecting onchocerciasis control: lessons for infection control. Expert Review Anti-infective Therapy 15: 377 - 386. Review

Cheke, R.A., Little, K.E., Young, S., et al., 2020. Taking the strain out of onchocerciasis? A reanalysis of blindness and transmission data does not support the existence of a savannah blinding strain of onchocerciasis in West Africa. Adv. Parasitol. (in review).

Choi ,Y.J., Tyagi, R., McNulty, S.N., et al., 2016. Genomic diversity in Onchocerca volvulus and its Wolbachia endosymbiont. Nat. Microbiol. 2:16207.

Colebunders, R., Nelson Siewe, F.J., Hotterbeekx, A. 2018. Onchocerciasis-associated epilepsy, an additional reason for strengthening onchocerciasis elimination programs. Trends Parasitol. 34, 208216. Review.

Comandatore, F., Cordaux, R., Bandi, C., et al., D. 2015. Supergroup C Wolbachia, mutualist symbionts of filarial nematodes, have a distinct genome structure. Open Biol. 5, 150099.

Connor, D.H., Gibson, D.W,, Neafie, R.C., et al., 1983. Sowda - onchocerciasis in north Yemen: a clinicopathologic study of 18 patients. Am. J Trop. Me.d Hyg. 32, 123-37.

Cotton, J.A., Bennuru, S., Grote, A., et al., 2016. The genome of Onchocerca volvulus, agent of river blindness. Nat. Microbiol. 2:16216.

Crainey, J.L., Wilson, M.D., Post, R.J. 2010. Phylogenetically distinct Wolbachia gene and pseudogene sequences obtained from the African onchocerciasis vector Simulium squamosum. Int. J. Parasitol 40, 569-578.

Crainey, J.L., da Silva, T.R.R., Encinas, F., et al., 2016. The mitogenome of Onchocerca volvulus from the Brazilian Amazonia focus. Mem. Inst. Oswaldo Cruz. 111, 79-81.

Crainey, J.L., Hurst, J., Lamberton, P.H.L., et al., 2017. The genomic architecture of novel Simulium damnosum Wolbachia prophage sequence elements and implications for onchocerciasis epidemiology. Frontiers in Microbiology 8: 852-869.

Crisp, G. 1956. Simulium and onchocerciasis in the northern territories of the Gold Coast. London, H.K.Lewis and Co. Ltd., pp. 171.

Crosskey, R.W.1990. The natural history of blackflies. John Wiley and Sons, Ltd, Chichester, UK, 711 pp.

Crump A, Morel CM, Omura S., 2012. The onchocerciasis chronicle: from the beginning to the end? Trends Parasitol. 28, 280-288.

Cupp, E.W., Sauerbrey, M., Richards, F. 2011. Elimination of human onchocerciasis: history of progress and current feasibility using ivermectin (Mectizan(@)) monotherapy. Acta Trop. 120 Suppl 1, S100-108.

Dadzie, Y., Neira, M., Hopkins, D. 2003. Final report of the Conference on the eradicability of onchocerciasis. Filaria J. 2, 2.

Dadzie, Y., Amazigo, U.V., Boatin, B.A., et al., 2018. Is onchocerciasis elimination in Africa feasible by 2025: a perspective based on lessons learnt from the African control programmes. Infect. Dis. Poverty. 7, 63.

Darge, K., Büttner, D.W. 1995. Ivermectin treatment of hyperreactive onchodermatitis (sowda) in Liberia. Trop Med Parasitol. 46, 206-212.

Davies, J.B. 1994. Sixty years of onchocerciasis vector control: a chronological summary with comments on eradication, reinvasion, and insecticide resistance. Annu. Rev. Entomol. 39, 23-45.

Demanou, M., Enyong, P., Pion, S.D.S., et al., 2003. Experimental studies on the transmission of Onchocerca volvulus by its vector in the Sanaga valley (Cameroon): Simulium squamosum B. Intake of microfilariae and their migration to the haemocoel of the vector. Ann. Trop. Med. Parasitol. , 97, 381-402.

Denery, J.R., Nunes, A.A., Hixon, M.S., et al., 2010. Metabolomics-based discovery of diagnostic biomarkers for onchocerciasis. PLoS. Negl. Trop. Dis. 4, pii: e834.

De Sole, G., Remme, J., Awadzi, K., et al., 1989. Adverse reactions after large-scale treatment of onchocerciasis with ivermectin: combined results from eight community trials. Bull. World Health Organ. 67, 707-719.

De Sole, G., Baker, R., Dadzie, K. Y., et al., 1991. Onchocerciasis distribution and severity in five West African countries. Bull. World Health Organ. 69, 689-698.

Diawara, L., Traore, M.O., Badji, A., et al., 2009. Feasibility of onchocerciasis elimination with ivermectin treatment in endemic foci in Africa: first evidence from studies in Mali and Senegal. PLoS Negl. Trop. Dis. 3, 497.

Dissak-Delon, F.N., Kamga, G.R., Humblet, P.C., et al., 2019. Docommunities really "direct" in communitydirected interventions? a qualitative assessment of beneficiaries' perceptions at 20 years of community directed treatment with ivermectin in Cameroon. Trop. Med. Infect. Dis. 4, 105.

Ditgen, D., Anandarajah, E.M., Meissner, K.A., et al., 2014. Harnessing the helminthsecretome for therapeutic immunomodulators. Biomed. Res. Int. 964350. Review. 
Doetze, A., Satoguina, J., Burchard, G., et al., 2000. Antigen-specificcellular hyporesponsiveness in a chronic human helminth infection is mediated by T(h)3/T(r)1-type cytokines IL-10 and transforming growth factor-beta but not by a T(h)1 to T(h)2 shift. Int Immunol. 12, 623-630.

Donelson, J.E., Duke, B.O.L., Moser, D., et al., 1988. Construction of Onchocerca volvulus cDNA libraries and partial characterization of the cDNA for a major antigen. Mol. Biochem. Parasitol. 31, 241-250.

Druet-Cabanac, M., Preux, P.M., Bouteille, B., et al., 1999. Onchocerciasis and epilepsy: a matched casecontrol study in the Central African Republic. Am. J. Epidemiol. 149, 565-570.

Duke, B.O.L, Lewis, D.J., Moore, P.J. 1966. Onchocerca-Simulium complexes. I. Transmission of forest and Sudan-savanna strains of Onchocerca volvulus, from Cameroon, by Simulium damnosum from various West African bioclimatic zones. Ann. Trop. Med. Parasitol. 60:318-326.

Duke, B.O.L. 1967a. Onchocerca-Simulium complexes. IV. Transmission of a variant of the forest strain of Onchocerca volvulus. Ann. Trop. Med. Parasitol. 61, 326-331.

Duke, B.O.L. 1967b. Infective filaria larvae other than Onchocerca volvulus in Simulium damnosum. Ann Trop. Med. Parasitol. 61, 700-705.

Duke, B.O.L. 1970. Onchocerca-Simulium complexes. VI. Experimental studies on the transmission of Venezuelan and West African strains of Onchocerca volvulus by Simulium metallicum and Simulium exiguum in Venezuela. Ann. Trop. Med. Parasitol. 64, 421-431.

Duke, B.O.L. 1972. Onchocerciasis. Br. Med. Bull. 28, 66-71. Review.

Duke, B.O.L. 1980. Observations on Onchocerca volvulus in experimentally infected chimpanzees (Pan troglotydes). Tropenmed. Parasit. 31. 41-54.

Duke, B.O.L. 1990. Human onchocerciasis--an overview of the disease. Acta Leiden. 59, 9-24. Review

Duke, B.O.L. 1998. Onchocerciasis, epilepsy and hyposexual dwarfism. Trans. R. Soc. Trop. Med. Hyg. 92, 236.

Eberle, R., Brattig, N.W., Trusch, M., et al., 2015. Isolation, identification and functional profile of excretorysecretory peptides from Onchocerca ochengi. Acta Trop. 142, 156-166.

Ehrens, A., Lunde, C.S., Jacobs, R.T., et al., 2020. In vivo efficacy of the boron-pleuromutilin an11251 against Wolbachia of the rodent filarial nematode Litomosoides sigmodontis. PLoS Negl. Trop. Dis. 14, e0007957.

Eisenbarth, A., Achukwi, M.D., Renz, A. 2016. Ongoing transmission of Onchocerca volvulus after 25 years of annual ivermectin mass treatments in the Vina du Nord river valley, in North Cameroon. PLoS Negl. Trop. Dis. 10, e0004392.

Erttmann, K.D., Unnasch, T.R., Greene, B.M., et al., 1987. A DNA sequence specific for forest form Onchocerca volvulus. Nature 327, 415-417.

Fain, A., Wery, M., Tilkin, J. 1981. Transmission of Onchocerca volvulus by Simulium albivirgulatum in the endemic area for onchocercosis of the Central Basin, Zaire. Ann. Soc. Belg. Med. Trop. 61, 307-309.

Figueroa Marroquin, H., Garcia Guillioli, C. 1971. Present status of Robles' disease in Guatemala. Rev. Invest. Salud Publica. 31, 17-25

Figueroa Marroquin, H. 1975. Die Robles' Krankheit (Onchocerciasis americana) und ihre Bedeutung in Guatemala. In: VIII. Tagung der Deutschen Tropenmedizinischen Gesellschaft anlässlich des 75jährigen Bestehens des Bernhard-Nocht-Instituts für Schiffs- und Tropenkrankheiten. Hamburg, 9.11. Oktober 1975

Fischer, P., Garms, R., Büttner, D.W., et al., 1997. Reduced prevalence of onchocerciasis in Uganda following either deforestation or vector control with DDT. East Afr. Med. J. 74, 321-325.

Frempong, K.K., Walker, M., Cheke, R.A., et al., 2016. Does increasing treatment frequency address s uboptimal responses to ivermectin for the control and elimination of river blindness? Clin. Infect. Dis. 62, 1338-1347.

Frentzel-Beyme, R. 1973. The prevalence of onchocerciasis and blindness in the population of the BongRange, Liberia. Z. Tropenmed. Parasit. 24, 339-357.

Frentzel-Beyme, R.R. 1975a. The geographical distribution of Onchocerca volvulus infection in Liberia. Tropenmed. Parasitol. 26, 70-87.

Frentzel-Beyme, R.R. 1975b. Visual impairment and incidence of blindness in Liberia and their relation to onchocerciasis. Tropenmed. Parasitol.26: 469-488.

Fülleborn, F. 1908. Ueber Filaria volvulus Leuckart. Arch. f. Schiffs- und Tropen-Hygiene, Beiheft 7 (Bd. XII.), $17 \mathrm{pp}$.

Fülleborn, F. 1924. The "blinding filarial" of Guatemala (Onchocerca caecutiens, Brumpt 1919). In Proceedings of the International Conference on Health Problems in Tropical Countries, pp. 241-255.

Gallin, M.Y., Jacobi, A.B., Büttner, D.W., et al., 1995. Human autoantibody to defensin: disease association with hyperreactive onchocerciasis (sowda). J. Exp. Med. 182, 41-47.

Gardon, J., Gardon-Wendel, N., Demanga-Ngangue, et al., 1997. Serious reactions after mass treatment of onchocerciasis with ivermectin in an area endemic for Loa loa infection. Lancet 350, 18-22. 
1313

1314

1315

1316

1317

1318

1319

1320

1321

1322

1323

1324

1325

1326

1327

1328

1329

1330

1331

1332

1333

1334

1335

1336

1337

1338

1339

1340

1341

1342

1343

1344

1345

1346

1347

1348

1349

1350

1351

1352

1353

1354

1355

1356

1357

1358

1359

1360

1361

1362

1363

1364

1365

1366

1367

1368

1369

1370

1371

1372

Garms, R., Post, A. 1966. Die Verbreitung von Simulium damnosum in Guinea/Westafrika. Z. Tropenmed. Parasitol. 17, 443-466.

Garms, R. 1972. Vorkommen phoretischer Simulien in Liberia. Z. Tropenmed. Parasitol. 23, 302-307.

Garms, R., Vajime, C.G. 1975a. On the ecology and distribution of the species of the Simulium damnosum complex in different bioclimatic zones of Liberia and Guinea. Tropenmed. Parasitol. 26, 375-380.

Garms, R. 1975b. Observations on filarial infections and parous rates of anthropophilic blackflies in in Guatemala, with reference to the transmission of Onchocerca volvulus. Tropenmed. Parasitol. 26, 169-182.

Garms, R. 1978. Use of morphological characters in the study of Simulium damnosum s.l. populations in West Africa. Tropenmed. Parasitol. 29, 483-491.

Garms, R., Ochoa J.0. 1979a. Further studies on the relative importance of Guatemalan blackfly species as vectors of Onchocerca volvulus. Tropenmed. Parasitol. 30, 120-128.

Garms, R., Walsh, J.F., Davies, J.B. 1979b. Studies on the reinvasion of the Onchocerciasis Control Programme in the Volta River Basin by Simulium damnosum s.l. with emphasis on the south-western areas. Tropenmed. Parasitol. 30, 345-362.

Garms, R., Cheke, R.A., Vajime, C.G., et al., 1982, The occurrence and movements of different members of the Simulium damnosum complex in Togo and Benin. Z. Angew. Zool., 69, 219-236.

Garms, R., Kerner, M. 1982. Anthropophily of Simulium damnosum s.l. and its rôle as a vector of human onchocerciasis in the Yemen Arab Republic. Tropenmed. Parasitol. 33, 175-180.

Garms, R., Cheke , R.A. 1985, Infections with Onchocerca volvulus in different members of the Simulium damnosum complex in Togo and Benin. Z. Angew. Zool. 72, 479-495.

Garms, R. 1987. Occurrence of the savanna species of the Simulium damnosum complex in Liberia. Trans. R. Soc. Trop. Med. Hyg. 81, 518.

Garms, R., Kerner M, Meredith, S.E.0. 1988. Simulium (Edwardsellum) rasyani n.sp., the Yemen species of the Simulium damnosum complex. Trop. Med. Parasitol. 39, 239-244.

Garms, R., Cheke, R.A., Sachs, R. 1991. A temporary focus of savanna species of the Simulium damnosum complex in the forest zone of Liberia. Trop. Med. Parasitol. 42, 181-187.

Garms, R, Yocha, J., Kipp, W. 1994. Decline of Simulium neavei and its associated crabs in the onchocerciasis foci of the Ruwenzori area, West Uganda, during the past 20 years. Brit. Simuliid Group Bull. 3, 11-12.

Garms, R., Lakwo, T.L., Ndyomugyenyi, R., et al., 2009. The elimination of the vector Simulium neavei from the Itwara onchocerciasis focus in Uganda by ground larviciding. Acta Trop 111, 203-210.

Garms, R., Badu, K.,Owusu-Dabo, E., et al., 2015. Assessments of the transmission of Onchocerca volvulus by Simulium sanctipauli in the Upper Denkyira District, Ghana, and the intermittent disappearance of the vector. Parasitol. Res 114, 1129-1137.

Garraud, O., Nkenfou, C., Bradley, J.E., et al., 1996. Differential regulation of antigen-specific IgG4 and IgE antibodies in response to recombinant filarial proteins. Int. Immunol. 8, 1841-1848.

Gasparini, G. 1962. "Sowda" a new disease or an unpublished type of onchocerciasis? Arch. Ital. Sci. Med. Trop. Parasitol. 43, 635-646.

Gebrezgabiher, G., Mekonnen, Z., Yewhalaw, D., et al., 2019.Reaching the last mile: main challenges relating to and recommendations to accelerate onchocerciasis elimination in Africa. Infect. Dis. Poverty. 8, 60. Review.

George, P.J., Hess, J.A., Jain, S., et al., 2019. Antibody responses against the vaccine antigens Ov-103 and Ovral-2 are associated with protective immunity to Onchocerca volvulus infection in both mice and humans. PLoS. Negl. Trop. Dis. 13, e0007730.

Globisch, D., Moreno, A.Y., Hixon, M.S., et al., 2013. Onchocerca volvulus-neurotransmitter tyramine is a biomarker for river blindness. Proc. Natl. Acad. Sci. U.S.A. 110, 4218-4223.

Gonzalez-Moa, M.J., Van Dorst, B., Lagatie, O., et al., 2018. Proof-of-Concept rapid diagnostic test for onchocerciasis: exploring peptide biomarkers and the use of gold nanoshells as reporter nanoparticles. ACS Infect. Dis. 4, 912-917.

Graham, S.P., Lustigman, S., Trees, A.J., et al., 2000. Onchocerca volvulus: Comparative analysis of antibody responses to recombinant antigens in two animal models of onchocerciasis. Exp. Parasitol. 94, 158-162.

Greene, B.M. 1987. Primate models for onchocerciasis research. Ciba Found Symp. 127, 236-243.

Güzelhan, C., Garms, R. 1991. Cytogenetic comparison of Simulium soubrense populations in Liberia (Simuliidae, Diptera). Z. angew. Zool. 78, 179-187.

Guillet, P., Escaffre, H., Ouedraogo, M., et al., 1980. Mise en évidence d'une résistance au téméphos dans le complexe Simulium damnosum [S. sanctipauli et $S$. soubrense] en Côte d'ivoire (Zone du programme de lutte contre l'onchocercose dans la région du Bassin de la Volta). Cahiers. O.R.S.T.O.M., sér. Ent. méd. et Parasitol. 18, 291-299. 
Hadis, M., Wilson, M.D., Cobblah, M., et al., 2005. Cytotaxonomic description of Simulium kaffaense, a new member of the S. damnosum complex (Diptera: Simuliidae) from south-western Ethiopia. Annals Trop. Med. Parasitol. 99, 267-291.

Haffner, A., Guilavogui, A.Z., Tischendorf, F.W., et al., 1998. Onchocerca volvulus: microfilariae secrete elastinolytic and males nonelastinolytic matrix-degrading serine and metalloproteases. Exp. Parasitol. 90, 26-33.

Hall, L.R., Pearlman, E. 1999. Pathogenesis of onchocercal keratitis (river blindness). Clin. Microbiol. Rev. 12, 445-453. Review

Hassan, A., Shaban, N. 2020. Onchocerciasis dynamics: modelling the effects of treatment,education and vector control. J. Biol. Dyn. 14, 245-268.

Hedtke, S.M., Kuesel, A.C., Crawford, K.E., et al., 2020. Genomic epidemiology in filarial nematodes: transforming the basis for elimination program decisions. Front Genet. 10, 1282. Review.

Hendy, A., Sluydts, V., Tushar, T., et al., 2017. Esperanza Window Traps for the collection of anthropophilic blackflies (Diptera: Simuliidae) in Uganda and Tanzania. PLoS Negl Trop Dis 11(6): e0005688.

Henkle-Dührsen K, Kampkötter A. 2001. Antioxidant enzyme families in parasitic nematodes. Mol Biochem Parasitol. 114, 129-142. Review

Hernández-González, A., Moya, L., Perteguer, M.J., et al., 2016. Evaluation of onchocerciasis seroprevalence in Bioko Island (Equatorial Guinea) after years of disease control programmes. Parasit. Vectors 9, 509.

Herrador, Z., Garcia, B., Ncogo, P., et al., 2018. Interruption of onchocerciasis transmission in Bioko Island: Accelerating the movement from control to elimination in Equatorial Guinea. PLoS Negl. Trop. Dis. 12 , e0006471.

Hertig, M., Wolbach, S.B. Studies on Rickettsia-like micro-organisms in Insects. 1924. J. Med. Res. 44, 329374.

Hewitson, J.P., Grainger, J.R., Maizels, R.M. 2009. Helminth immunoregulation: The role of parasite secreted proteins in modulating host immunity. Mol. Biochem. Parasitol. 167, 1-11.

Higazi, T.B., Katholi, C.R., Mahmoud, B.M., et al., 2001. Onchocerca volvulus: genetic diversity of parasite isolates from Sudan. Exp. Parasitol. 97, 24-34.

Higazi, T.B., Zarroug, I.M., Mohamed, H.A., et al., 2013. Interruption of Onchocerca volvulus transmission in the Abu Hamed focus, Sudan. Am. J. Trop. Med. Hyg. 89, 51-57.

Hirai, H., Tada, I., Takahashi, H., et al., 1987. Chromosomes of Onchocerca volvulus (Spirurida: Onchocercidae): a comparative study between Nigeria and Guatemala. J. Helminthol. 61, 43-46.

Hoerauf, A., Volkmann, L., Hamelmann, C., et al., 2000.Endosymbiotic bacteria in worms as targets for a novel chemotherapy in filariasis. Lancet 355, 1242-1243.

Hoerauf, A., Mand, S., Adjei, O., et al., 2001. Depletion of Wolbachia endobacteria in Onchocerca volvulus by doxycycline and microfilaridermia after ivermectin treatment. Lancet 357, 1415-1416.

Hoerauf, A., Kruse, S., Brattig, N.W., et al., 2002. The variant Arg110Gln of human IL-13 is associated with an immunologically hyper-reactive form of onchocerciasis (sowda). Microbes Infect. 4, 37-42.

Hoerauf, A., Brattig, N. 2002. Resistance and susceptibility in human onchocerciasis - beyond Th1 vs. Th2. Trends Parasitol. 18, 25-31. Review

Hoerauf, A., Büttner, D.W., Adjei, O., et al., 2003a. Onchocerciasis Brit. Med. J., 326, 207-210. Review.

Hoerauf, A., Mand, S., Volkmann, L., et al., 2003b. Doxycycline in the treatment of human onchocerciasis: Kinetics of Wolbachia endobacteria reduction and of inhibition of embryogenesis in female Onchocerca worms. Microbes Infect. 5, 261-273.

Hoerauf, A., Specht, S., Büttner, M., et al., 2008. Wolbachia endobacteria depletion by doxycycline as antifilarial therapy has macrofilaricidal activity in onchocerciasis: a randomized placebo-controlled study. Med. Microbiol. Immunol. 197, 295-311.

Hoffmann, A.A., Montgomery, B.L., Popovici, J., I et al., 2011. Successful establishment of Wolbachia in Aedes populations to suppress dengue transmission. Nature 476, 454-459.

Hong, W.D., Benayoud, F., Nixon, G.L., et al., 2019. AWZ1066S, a highly specific anti-Wolbachia drug candidate for a short-course treatment of filariasis. Proc. Natl. Acad. Sci. U.S.A. 116, 1414-1419.

Hopkins, A.D. 2016. Neglected tropical diseases in Africa: a new paradigm. Int Health 8 (Suppl 1), i28-i33.

Hotez, P.J., Alvarado, M., Basáñez, M.G., et al., 2014. The global burden of disease study 2010: interpretation and implications for the neglected tropical diseases. PLoS Negl. Trop. Dis. 8, e2865.

Hotez, P.J., Bottazzi, M.E., Zhan, B., et al., 2015. The Onchocerciasis Vaccine for Africa--TOVA-Initiative. PLoS Negl. Trop. Dis. 9, e0003422.

Hougard, J. M., Yaméogo, L., Sékétéli, A., et al., 1997. Twenty-two years of blackfly control in the onchocerciasis control programme in West Africa. Parasitol. Today 13, 425-431. 
1431

1432

1433

1434

1435

1436

1437

1438

1439

1440

1441

1442

1443

1444

1445

1446

1447

1448

1449

1450

1451

1452

1453

1454

1455

1456

1457

1458

1459

1460

1461

1462

1463

1464

1465

1466

1467

1468

1469

1470

1471

1472

1473

1474

1475

1476

1477

1478

1479

1480

1481

1482

1483

1484

1485

1486

1487

1488

1489

Jacob, B.G., Loum, D., Lakwo, T.L., et al., 2018. Community directed vector control to supplement mass drug distribution for onchocercerciasis elimination in the Madi mid-North focus in Northern Uganda. PLOS Negl.Trop. Dis. 2018.

Jaurigue, J.A., Seeberger, P.H. 2017. Parasite carbohydrate vaccines. Front. Cell. Infect. Microbiol. 7, 248.

Johnson, T.P., Tyagi, R., Lee, P.R., et al., 2017. Nodding syndrome may be an autoimmune reaction to the parasitic worm Onchocerca volvulus. Sci. Transl. Med. 9, 377.

Kahl, J., Brattig, N., Liebau, E. 2018. The untapped pharmacopeic potential of helminths. Trends Parasitol. 34, 828-842. Review.

Kamga, G.-R., Dissak-Delon, F.N., Nana-Djeunga, H.C., et al., 2016. Still mesoendemic onchocerciasis in two Cameroonian community-directed treatment with ivermectin projects despite more than 15 years of mass treatment. Parasit. Vectors 9, 581.

Kamga, G.-R., Dissak-Delon, F.N., Nana-Djeunga, H.C., et al., 2017. Important progress towards elimination of onchocerciasis in the West Region of Cameroon. Parasit. Vectors, 10, 373.

Kashan, A., Garms, R. 1987. Cytotaxonomy of the Simulium sanctipauli sub-complex in Liberia. Trop. Med. Parasit. 38, 289-293.

Katabarwa, M.N., Eyamba, A., Nwane, P., et al., 2013. Fifteen years of annual mass treatment of onchocerciasis with ivermectin have not interrupted transmission in the West region of Cameroon. J. Parasitol. Res. 2013, 420928.

Katabarwa, M., Lakwo, T., Habomugisha, P., et al., 2014a. Transmission of Onchocerca volvulus by Simulium neavei in Mount Elgon focus of eastern Uganda has been interrupted. Am. J. Trop. Med. Hyg. 90, 11591166.

Katabarwa, M.N., Endeshaw, T., Taye, A., et al., 2014b. The disappearance of onchocerciasis without intervention in Tigray Region in northwest Ethiopia. Pathog. Glob. Health..108, 123.

Katabarwa, M.N., Katamanywa, J., Lakwo, T., et al., 2016. The Imaramagambo onchocerciasis focus in southwestern Uganda: interruption of transmission after disappearance of the vector Simulium neavei and its associated freshwater crabs. Am. J. Trop. Med. Hyg. 95, 417- 425.

Katabarwa, M.N., Lakwo, T., Habomugisha, P., et al., 2018. After 70 years of fighting an age-old scourge, onchocerciasis in Uganda, the end is in sight. Int. Health 10 (suppl 1), i79-i88.

Katabarwa, M.N., Habomugisha, P., Khainza, A., et al., 2020a. Historical Elimination of Onchocerciasis from Victoria Nile focus in central Uganda verified using Who criteria. Am. J. Trop. Med. Hyg. 2020 Mar 30.

Katabarwa, M.N., Zarroug, I.M.A., Negussu, N., et al., 2020b. The Galabat-Metema cross-border onchocerciasis focus: The first coordinated interruption of onchocerciasis transmission in Africa. PLoS Negl. Trop. Dis. 14, e0007830

Katawa, G., Layland, L.E., Debrah, A.Y., et al., 2015. Hyperreactive onchocerciasis is characterized by a combination of Th17-Th2 immune responses and reduced regulatory T cells. PLoS Negl. Trop. Dis. 9, e3414.

Kazura, J.W. 2016. Onchocerciasis elimination from Africa: One step in Northern Sudan. Am. J. Trop. Med. Hyg. 95, 983-984.

Kim, Y.E., Remme, J.H., Steinmann, P., et al., 2015. Control, elimination, and eradication of river blindness: scenarios, timelines, and ivermectin treatment needs in Africa. PLoS Negl. Trop. Dis. 9, e0003664.

Knight, A. 2008. The beginning of the end for chimpanzee experiments? Philos. Ethics Humanit. Med. 3, 16.

Knüttgen, H-J. 1964. Untersuchungen über Vorkommen und Bedeutung der Onchozerkose in Guinea, Westafrika. Z. Tropenmed. Parasit. 15, 427-435.

Knüttgen, H.J., Büttner, D.W. 1968. Untersuchungen zur Epidemiologie und Bedeutung der Onchozerkose in Oberguinea. Z. Trop. Med. Parasitol. 19, 1- 42.

Knüttgen, H.J. 1971. Remarks on the epidemiology and importance of onchocerciasis in Upper Guinea. Ann. Soc. Belg. Med. Trop. 51, 611-614.

Korten, S., Wildenburg, G., Darge, K., et al., 1998. Mast cells in onchocercomas from patients with hyperreactive onchocerciasis (sowda). Acta Trop. 70, 217-231.

Korten, S., Badusche, M., Büttner, D.W., et al., 2008. Natural death of adult Onchocerca volvulus and filaricidal effects of doxycycline induce local FOXP3+/CD4+ regulatory T cells and granzyme expression. Microbes Infect. 10, 313-324.

Korten, S., Hoerauf, A., Kaifi, J.T., et al., 2011. Low levels of transforming growth factor-beta (TGF- beta) and reduced suppression of Th2-mediated inflammation in hyperreactive human onchocerciasis. Parasitology 138, 35-45.

Kozek, W.J., Figueroa-Marroquin, H.F. 1977. Intracytoplasmic bacteria in Onchocerca volvulus. Am. J. Trop. Med. Hyg. 26:663-678.

Krüger, A., Nurmi, V., Yocha, J., et al., 1999. The Simulium damnosum complex in western Uganda and its role as a vector of Onchocerca volvulus. Trop. Med. Int. Health 4, 819-826. 
Kuesel, A.C. 2016. Research for new drugs for elimination of onchocerciasis in Africa. Int. J. Parasitol. Drugs. Drug Resist. 6, 272-286.

Kurtak, D.C., Meyer, R., Ocran, M., et al., 1987. Management of insecticide resistance in control of the Simulium damnosum complex by the Onchocerciasis Control Programme, West Africa: potential use of negative correlation between organophosphate resistance and pyrethroid susceptibility. Med. Vet. Entomol. 1, 137-146.

Kutin, K., Kruppa, T.F., Brenya, R., et al., 2004. Efficiency of Simulium sanctipauli as a vector of Onchocerca volvulus in the forest zone of Ghana. Med. Vet. Ent. 18, 167-173.

Lagatie, O., Merino, M., Batsa Debrah, L., et al., 2016. An isothermal DNA amplification method for detection of Onchocerca volvulus infection in skin biopsies. Parasit. Vectors. 9, 624.

Lagatie, O., Verheyen, A., Van Dorst, B., et al., 2018. Linear epitopes in Onchocerca volvulus vaccine candidate proteins and excretory-secretory proteins. Parasite Immunol. 40, e12587.

Lagatie, O., Verheyen, A., Nijs, E., et al., 2019. Performance evaluation of three serodiagnostic peptide epitopes and the derived multi-epitope peptide OvNMP-48 for detection of Onchocerca volvulus infection. Parasitol. Res. 118, 2263-2270.

Lakwo,T.L., Garms, R., Wamani, J., et al., 2017. Interruption of the transmission of Onchocerca volvulus in the Kashoya-Kitomi focus, western Uganda by long-term ivermectin treatment and elimination of he vector Simulium neavei by larviciding. Acta Trop. 167, 128-136.

Lamberton, P.H.L., Cheke, R.A., Winskill, P., et al., 2015. Onchocerciasis transmission in Ghana: persistence under different control strategies and the role of the simuliid vectors. PLoS. Negl.Trop. Dis. 9(4), e0003688.

Lefoulon, E., Giannelli, A., Makepeace, B.L., et al., 2017. Whence river blindness? The domestication of mammals and host-parasite co-evolution in the nematode genus Onchocerca. Int. J. Parasitol. 47, 457470.

Leuckart, R. 1893. Filaria volvuloxus. in Manson P., Sir Patrick, 1903: Diseases of the skin in tropical climates, in Davidson's Textbook of Hygiene and Diseases of warm climates. Tropical Diseases, LONDON, 628-995.

Lewis, D.J., 1953. Simulium damnosum and its relation to onchocerciasis in the Anglo-Egyptian Sudan. Bull. Entomol. Res. 43, 597-564.

Lewis, D.J., Duke, B.O.L., 1966. Onchocerca-Simulium complexes. II. Variation in West African female Simulium damnosum. Ann. Trop. Med. Parasit. 60, 337-346.

Little, M.P., Basáñez, M.-G., Breitling, L.P., et al., 2004. Incidence of blindness during the Onchocerciasis Control Programme in western Africa, 1971-2002. J. Infect. Dis. 189, 1932-1941.

Lloyd, M.M., Gilbert, R., Taha, N.T., et al., 2015. Conventional parasitology and DNA-based diagnostic methods for onchocerciasis elimination programmes. Acta Trop. 146, 114-118.

Lobos, E., Weiss, N., Karam, M., et al., 1991. An immunogenic Onchocerca volvulus antigen: a specific and early marker of infection. Science 251, 1603-1605.

Lont, Y.L., Coffeng, L.E., de Vlas, S.J., et al., 2017. Modelling anti-Ov16 IgG4 antibody prevalence as an indicator for evaluation and decision making in onchocerciasis elimination programmes. PLoS. Negl. Trop. Dis. 11, e0005314.

Lucius, R., Büttner, D.W., Kirsten, C., et al., 1986. A study on antigen recognition by onchocerciasis patients with different clinical forms of disease. Parasitology 92, 569-580

Lucius, R., Schulz-Key, H., Büttner, D.W., et al., 1988. Characterization of an immunodominant Onchocerca volvulus antigen with patient sera and a monoclonal antibody. J. Exp. Med. 167, 1505-1510.

Lugga, M.C.L., Chane, F., 2011. Onchocerciasis control in South Sudan. South Sudan Medical Journal 4: 6162.

Lustigman, S., James, E.R., Tawe, W., et al., 2002. Towards a recombinant antigen vaccine against Onchocerca volvulus. Trends Parasitol. 18, 135-141. Review.

Lustigman, S., MacDonald, A.J., Abraham, D. 2003. CD4+-dependent immunity to Onchocerca volvulus thirdstage larvae in humans and the mouse vaccination model: common ground and distinctions. Int. J. Parasitol. 33, 1161-1171. Review.

Lustigman, S., Makepeace, B.L., Klei, T.R., et al., 2018. Onchocerca volvulus: the road from basic biology to a vaccine. Trends Parasitol. 2018 Jan;34(1):64-79.

Macfarlane, C.L., Quek, S., Pionnier, N., et al., 2020. The insufficiency of circulating miRNA and DNA as diagnostic tools or as biomarkers of treatment efficacy for Onchocerca volvulus. Sci Rep. 10, 6672.

Mackenzie, C.D., Williams, J.F., Sisley, B.M., et al., 1985. Variations in host responses and the pathogenesis of human onchocerciasis. Rev. Infect. Dis. 7, 802-808.

Mahdy, M.A.K., Abdul-Ghani, R., Abdulrahman, T.A.A., et al., 2018. Onchocerca volvulus infection in the Tihama region - west of Yemen: continuing transmission in ivermectin-targeted endemic foci and unveiled endemicity in districts with previously unknown status. PLoS Negl. Trop. Dis. 12, e0006329. 
Maizels, R.M., Smits, H.H., McSorley, H.J., 2018. Modulation of host immunity by helminths: the expanding repertoire of parasite effector molecules. Immunity 49, 801-818.

Makepeace, B.L., Tanya, V.N., 2016. 25 Years of the Onchocerca ochengi Model. Trends Parasitol. 32, 966978. Review.

Manchang, T.K., Ajonina-Ekoti, I., Ndjonka, D., et al., 2015. Immune recognition of Onchocerca volvulus proteins in the human host and animal models of onchocerciasis. J. Helminthol. 89, 375-386.

Mazzotti, L., 1951. Observations on the use of hetrazan in onchocerciasis in Mexico. Am. J. Trop. Med. Hyg. 31, 628-632.

McCrae, A.W.R. 1978. Intermittent eradication of Simulium damnosum Theo. on the Nile from Jinja, Uganda: 1951-1977. Med. Entom. Centenary Symposium Proceedings, 1978, 133-134.

McMahon, J.P., Highton, R.B., Goiny, H. 1958. The eradication of Simulium neavei from Kenya. Bull. World Health Org. 19, 75-107.

McNulty, S.N., Foster, J.M., Mitreva, M., et al., 2010. Endosymbiont DNA in endobacteria-free filarial nematodes indicates ancient horizontal genetic transfer. PLoS One. 5, e11029.

McNulty, S.N., Rosa, B.A., Fischer, P.U., et al., 2015. An integrated multiomics approach to identify candidate antigens for serodiagnosis of human onchocerciasis. Mol. Cell Proteomics. 14, 3224-3233.

Medina-De la Garza, C.E., Brattig, N.W., Tischendorf, F.W., et al., 1990. Serum-dependent interaction of granulocytes with Onchocerca volvulus microfilariae in generalized and chronic hyper-reactive onchocerciasis and its modulation by diethylcarbamazine. Trans. R. Soc. Trop. Med. Hyg. 84, 701-706.

Meredith, S.E.O., Cheke, R.A., Garms, R., 1983. Variation and distribution of forms of Simulium soubrense and S. sanctipauli in West Africa. Ann. Trop. Med. Parasitol. 77, 627-640.

Meyer, C.G., Gallin, M., Erttmann, K.D., et al., 1994. HLA-D alleles associated with generalized disease, localized disease, and putative immunity in Onchocerca volvulus infection. Proc. Natl. Acad. Sci. U S A. 91, 7515-7519.

Michael, E., Smith, M.E., Singh, B.K., et al., 2020. Data-driven modelling and spatial complexity supports heterogeneity-based integrative management for eliminating Simulium neavei-transmitted river blindness. Sci. Rep. 10, 4235.

Monahan, C.M., Chapman, M.R., French, D.D., et al., 1995. Efficacy of moxidectin oral gel against Onchocerca cervicalis microfilariae. J. Parasitol. 81, 117-118.

Morales-Hojas, R., Cheke, R.A., Post, R.J. 2006. Molecular systematics of five Onchocerca species (Nematoda: Filarioidea) including the human parasite, $O$. volvulus, suggest sympatric speciation. J. Helminthol. 80, 281-290.

Morales-Hojas, R., Cheke, R.A., Post, R.J. 2007. A preliminary analysis of the population genetics and molecular phylogenetics of Onchocerca volvulus (Nematoda: Filarioidea) using nuclear ribosomal second internal transcribed spacer sequences. Mem. Inst. Oswaldo Cruz, Rio de Janeiro, 102, 879-882.

Moya, L., Herrador, Z., Ta-Tang, T.H., et al., 2016. Evidence for suppression of onchocerciasis transmission in Bioko Island, Equatorial Guinea. Plos. Negl Trop Dis. 2016;10:e0004829.

Mpagi, J.L., Büttner, D.W., Tischendorf, F.W., et al., 2000. Use of the recombinant Onchocerca volvulus protein Ov20/OvS1 for the immunodiagnostic differentiation between onchocerciasis and mansonelliasis and for the characterization of hyperreactive onchocerciasis (sowda). Trop. Med. Int. Health 5, 891-897.

Navarro, M., Camprubí, D., Requena-Méndez, A., et al., 2020. Safety of high-dose ivermectin: a systematic review and meta-analysis. J. Antimicrob. Chemother. 75, 827-834.

Ndyomugyenyi, R. 1998. The burden of onchocerciasis in Uganda. Ann. Trop. Med. Parasit. 92 (Suppl. 1), 133-137.

Neumann, E., Lucasse, C., Gunders, A. 1964. Experimental onchocercal ocular lesions in the chimpanzee. Am. J. Ophthalmol. 57, 217-227.

Njume, F.N., Ghogomu, S.M., Shey, R.A., et al., 2019. Identification and characterization of the Onchocerca volvulus excretory secretory product Ov28CRP, a putative GM2 activator protein. PLoS Negl Trop Dis. 13, e0007591.

NTD Modelling Consortium Onchocerciasis Group. 2019. The World Health Organization 2030 goals for onchocerciasis: Insights and perspectives from mathematical modelling: NTD Modelling Consortium Onchocerciasis Group. Gates Open Res. 3, 1545.

Nutman, T.B., Steel, C., Ward, D.J., et al., 1991. Immunity to onchocerciasis: recognition of larval antigens by humans putatively immune to Onchocerca volvulus infection. J. Infect. Dis. 163, 1128-1133.

O'Day, J., Mackenzie, C.D. 1985. Ocular onchocerciasis. Diagnosis and current clinical approaches. Trop Doct. 15, 87-94. Review.

O'Hanlon, S.J., Slater, H.C., Cheke, R.A., et al., 2016. Model-based geostatistical mapping of the prevalence of Onchocerca volvulus in West Africa. PLoS Neglected Tropical Diseases 10: e0004328. 
Omar, M.S., Garms, R. 1975. The fate and migration of microfilariae of a Guatemalan strain of Onchocerca volvulus in Simulium ochraceum and S. metallicum, and the role of the buccopharyngeal armature in the destruction of microfilariae. Tropenmed. Parasitol. 26, 183-190.

Omar, M.S., Garms, R.,1977. Lethal damage to Simulium metallicum following high intakes of Onchocerca volvulus microfilariae in Guatemala. Tropenmed. Parasitol. 28, 109-119.

Omar, M.S., Franz, M., Büttner, D.W., 1979. Some observations on onchocerciasis including sowda in the Yemen Arab Republic. Tropenmed Parasitol. 30, 113-119.

Ōmura, S. 2016. Splendid gift from the Earth: the origins and impact of the Avermectins (Nobel Lecture). Angew. Chem. Int. Ed. Engl. 55, 10190-10209. Review.

O'Neill, J., 1875. On the presence of a filaria in "Craw craw". Lancet 1, 265-266.

Opoku, N.O., Bakajika, D.K., Kanza, E.M., et al., 2018. Single dose moxidectin versus ivermectin for Onchocerca volvulus infection in Ghana, Liberia, and the Democratic Republic of the Congo: a randomised, controlled, double-blind phase 3 trial. Lancet 392, 1207-1216.

Osei-Atweneboana, M.Y., Eng, J.K.L., Boakye, D.A., et al., 2007. Prevalence and intensity of Onchocerca volvulus infection and efficacy of ivermectin in endemic communities in Ghana: a two-phase epidemiological study. Lancet 369, 2021-2029.

Osei-Atweneboana, M.Y., Awadzi, K., Attah, S.K., et al., 2011. Phenotypic evidence of emerging ivermectin resistance in Onchocerca volvulus. PLoS Negl Trop Dis 5, e998.

Ottesen, E.A., 1995. Immune responsiveness and the pathogenesis of human onchocerciasis. J. Infect Dis. 171, 659-671. Review.

Painter, T.S., Griffen, A.B., 1937. The structure and the development of the salivary gland chromosomes of Simulium. Genetics 22, 612-633.

Parsons, A.C., 1908. Filaria volvulus Leuckart, its distribution, structure and pathological effects. Parasitology 1, 359-368.

Pearlman, E., 1997. Immunopathology of onchocerciasis: a role for eosinophils in onchocercal dermatitis and keratitis. Chem. Immunol. 66, 6-40. Review.

Pearlman, E., Gillette-Ferguson, I. 2007. Onchocerca volvulus, Wolbachia and river blindness. Chem. Immunol. Allergy 92, 254-265. Review.

Philippon, B., Remme, J.H., Walsh, J.F., et al., 1990. Entomological results of vector control in the Onchocerciasis Control Programme. Acta Leiden. 59, 79-94.

Picq, J.J., Coz, J., Jardel, J.P. 1971. A method of evaluating the density of the microfilaria of Onchocerca volvulus Leuckart, 1893 in patients with onchocerciasis: technic and reading time of skin biopsies. Bull. World Health Organ. 45, 517-520.

Plaisier, A.P., Van Oortmarssen, G.J., Habbema, J.D.F., et al. 1990. ONCHOSIM: a model and computer simulation program for the transmission and control of onchocerciasis. Comput. Methods Programs Biomed. 31, 43-56.

Plier, D.A., Awadzi, K., Freedman, D.O. 1995. Immunoregulation in onchocerciasis: persons with ocular inflammatory disease produce a Th2-like response to Onchocerca volvulus antigen. J. Infect. Dis. 174, 380-386.

Post, R.J. ,1986. The cytotaxonomy of Simulium sanctipauli and Simulium soubrense (Diptera: Simuliidae). Genetica 69, 191-207.

Post, R.J., McCall, P.J., Trees, A.J., et al., 1989. Chromosomes of six species of Onchocerca (Nematoda: Filarioidea). Trop. Med. Parasitol. 40, 292-294.

Post, R.J., Cheke, R.A., Boakye, D.A., et al., 2013. Stability and change in the distribution of cytospecies of the Simulium damnosum complex (Diptera: Simuliidae) in southern Ghana from 1971 to 2011. Parasites and Vectors 6, 205.

Prince, A.M., Brotman, B., Johnson, E.H. Jr., et al., 1992. Onchocerca volvulus: immunization of chimpanzees with X-irradiated third-stage (L3) larvae. Exp. Parasitol. 74: 239-250.

Puente, S., Ramirez-Olivencia, G., Lago, M., et al., 2018. Dermatological manifestations in onchocerciasis: a retrospective study of 400 imported cases. Enferm. Infecc. Microbiol. Clin. 36, 633-639.

Quintana, J.F., Makepeace, B.L., Babayan, S.A., et al., 2015. Extracellular Onchocerca-derived small RNAs in host nodules and blood. Parasit. Vectors. 8, 58.

Railliet, A., Henry, A.C., 1910. Remarques a` l'occasion de la note de. M. le Dr. Antoine. Bull. Soc. Pathol. Exot. Filiales 3, 91-93.

Rebollo, M.P., Zoure, H., Ogoussan, K., et al., 2018. Onchocerciasis: shifting the target from control to elimination requires a new first-step-elimination mapping. Int. Health. 10 (suppl_1), i14-i19.

Remme, J., Baker, R.H.A., De Sole, G., et al., 1989. A community trial of ivermectin in the onchocerciasis focus of Asubende, Ghana. I. Effect on the microfilarial reservoir and transmission of Onchocerca volvulus. Trop. Med. Parasit. 40, 367-374. 
Renz, A., 1987. Studies on the dynamics of transmission of onchocerciasis in a Sudan-savanna area of north Cameroon. III. Infection rates of the Simulium vectors and Onchocerca volvulus transmission potentials. Ann. Trop. Med. Parasitol. 81, 239-252.

Renz, A., Wenk, P., 1987. Studies on the dynamics of transmission of onchocerciasis in a Sudan savanna area of North Cameroon. I. Prevailing Simulium vectors, their biting rates and age-composition at different distances from their breeding sites. Ann. Trop. Med. Parasitol. 81, 215-228.

Richard-Lenoble, D., al Qubati, Y., Toe, L., et al., 2001. Human onchocerciasis and "sowda" in the Republic of Yemen. Bull. Acad. Natl. Med. 185, 1447-1459.

Richards, F.O., Eigege, A., Umaru, J., et al., 2020. The interruption of transmission of human onchocerciasis by an annual mass drug administration program in plateau and Nasarawa States, Nigeria. Am. J. Trop. Med. Hyg. 102, 582-592.

Robles, R., 1917. Enfermedad nueva en Guatemala. La Juventud Médica 17, 97-115.

Rodríguez-Pérez, M.A., Adeleke, M.A., Burkett-Cadena, N.D., et al., 2013. Development of a novel trap for the collection of black flies of the Simulium ochraceum complex. PLOS One. 2013;8(10):e76814. Epub 2013/10/12. pmid:24116169;

Rodríguez-Pérez, M.A., Fernández-Santos, N.A., Orozco-Algarra, M.E., et al., 2015. Elimination of onchocerciasis from Mexico. PLoS Negl Trop Dis. 9, e0003922.

Rolland, A., Prost, A., Thylefors, B., 1980. Review, after 3 years of the treatment with suramin, of a village suffering from onchocerciasis under entomological protection. Rev. Int. Trach. Pathol. Ocul. Trop. Subtrop. 57, 99-106.

Routledge, I., Walker, M., Cheke, R.A., et al., 2018. Modelling the impact of larviciding on the population dynamics and biting rates of Simulium damnosum (s.l.): implications for vector control as a complementary strategy for onchocerciasis elimination in Africa. Parasites and Vectors 11: 316.

Rubio de Krömer, M.T., Medina-De la Garza, C.E., Brattig, N.W., 1995. Differences in eosinophil and neutrophil chemotactic responses in sowda and generalized form of onchocerciasis. Acta Trop. 60, 21-33.

Ruiz Reyes, F. 1951. Treatment of onchocerciasis with diethylcarbamazine. Rev. Asoc. Medica. Mex. 31, 495-504.

Saint André A.v., Blackwell, N.M., Hall, L.R., et al., 2002. The role of endosymbiotic Wolbachia bacteria in the pathogenesis of river blindness. Science 295, 1892-1895.

Samba, E.M., 1994 The onchocerciasis control programme in West Africa. An example of effective public health management, WHO 1994 (1994, Public Health in Action 1), Geneva.

Sauerbrey, M., Rakers, L.J., Richards, F.0., 2018. Progress toward elimination of onchocerciasis in the Americas. Int. Health. 10(suppl_1), i71-i78.

Schönemeyer, A., Lucius, R., Sonnenburg, B., et al., 2001. Modulation of human T cell responses and macrophage functions by onchocystatin, a secreted protein of the filarial nematode Onchocerca volvulus. J. Immunol. 167, 3207-3215.

Schulz-Key, H., Karam, M. 1986. Periodic reproduction of Onchocerca volvulus. Parasitol Today. 2, 284-286. Seeber, F., Brattig, N., Soboslay, P.T., et al., 1993. Characterization of a recombinant T cell and B cell reactive polypeptide of Onchocerca volvulus. J Immunol. 150, 2931-294.

Shelley AJ, Hernández LM, Maia-Herzog M, et al. 2010.The blackflies (Diptera: Simuliidae) of Brazil. In: Arias JR, Golovatch S, Wantzen KM, et al., editors, Aquatic Biodiversity in Latin America (ABLA). Vol.6. Sofia-Moscow: Pensoft; $821 \mathrm{pp}$.

Shey, R.A., Ghogomu, S.M., Esoh, K.K., et al., 2019. In-silico design of a multi-epitope vaccine candidate against onchocerciasis and related filarial diseases. Sci Rep. 9, 4409

Siddiqui, M.A., al-Khawajah, M.M. 1991. The black disease of Arabia, Sowda-onchocerciasis. New findings. Int. J. Dermatol. 30, 130-133

Sironi, M., Bandi, C., Sacchi, L., et al., 1995. Molecular evidence for a close relative of the arthropod endosymbiont Wolbachia in a filarial worm. Mol. Biochem. Parasitol. 74:223-227.

Slatko, B.E., O'Neill, S.L., Scott, A.L., et al., 1999. The Wolbachia Genome Consortium. Microb. Comp. Genomics 4, 161-165.

Slatko, B.E., Taylor, M., Foster, J.M. 2010. The Wolbachia endosymbiont as an anti-filarial nematode target. Symbiosis 51, 55-65.

Smith, M.E., Bilal, S., Lakwo, T.L., et al., 2019. Accelerating river blindness elimination by supplementing MDA with a vegetation "slash and clear" vector control strategy: a data-driven modeling analysis. Sci Rep. 2019 Oct 24;9(1):15274.

Soboslay, P.T., Dreweck, C.M., Taylor, H.R., et al., 1991. Experimental onchocerciasis in chimpanzees. Cellmediated immune responses, and production and effects of IL-1 and IL-2 with Onchocerca volvulus infection. J. Immunol. 147:346-53. 
Soboslay, P.T., Lüder, C.G., Riesch, S., et al., 1999. Regulatory effects of Th1-type (IFN-gamma, IL-12) and Th2-type cytokines (IL-10, IL-13) on parasite-specific cellular responsiveness in Onchocerca volvulusinfected humans and exposed endemic controls. Immunology 97, 219-225.

Steel, C., Golden, A., Stevens, E., et al., 2015. Rapid point-of-contact tool for mapping and integrated surveillance of Wuchereria bancrofti and Onchocerca volvulus infection. Clin. Vaccine Immunol. 22, 896-901.

Steisslinger, V., Korten, S., Brattig, N.W., et al., 2015. DNA vaccine encoding the moonlighting protein Onchocerca volvulus glyceraldehyde-3-phosphate dehydrogenase (Ov-GAPDH) leads to partial protection in a mouse model of human filariasis. Vaccine 33, 5861-5867.

Strote, G., Brattig, N.W., Tischendorf, F.W., 1990. Ultrastructural study of the interaction between eosinophilic granulocytes and third and fourth stage larvae of Onchocerca volvulus. Acta Trop. 48, 1-8.

Ta, T.-H., Moya, L., Nguema, J., et al., 2018. Geographical distribution and species identification of human filariasis and onchocerciasis in Bioko Island, Equatorial Guinea. Acta Tropica 180, 12-17.

Takaoka, H., 2015. Review of the biology and ecology of adult blackflies in relation to the transmission of onchocerciasis in Guatemala. Trop. Med. Health. 43 (Suppl), 71-85.

Tamarozzi, F., Turner, J.D., Pionnier, N., et al., 2016. Wolbachia endosymbionts induce neutrophil extracellular trap formation in human onchocerciasis. Sci. Rep. 6, 35559.

Taylor, C.M., Wang, Q., Rosa, B.A., et al., 2013. Discovery of anthelmintic drug targets and drugs using chokepoints in nematode metabolic pathways. PLoS. Pathog. 9, e1003505.

Taylor, H.R., Trpis, M., Cupp, E.W., et al., 1988. Ivermectin prophylaxis against experimental Onchocerca volvulus infection in chimpanzees. Am. J. Trop. Med. Hyg. 39, 86-90.

Taylor, M.J., Hoerauf, A. 1999. Wolbachia bacteria of filarial nematodes. Parasitol. Today 15: 437-442. Review.

Taylor, M.J., Hoerauf, A., 2001. A new approach to the treatment of filariasis. Curr. Opin. Infect. Dis. 14, 727-731. Review.

Taylor, M.J., Awadzi, K., Basáñez, M.-G., et al., 2009. Onchocerciasis control: vision for the future from a Ghanaian perspective. Parasit. Vectors. 2, 7.

Taylor, M.J., Hoerauf, A., Bockarie, M. 2010. Lymphatic filariasis and onchocerciasis. Lancet 376, 11751185. Review.

Taylor, M.J., Hoerauf, A., Townson, S., et al., 2014. Anti-Wolbachia drug discovery and development: safe macrofilaricides for onchocerciasis and lymphatic filariasis. Parasitology 141, 119-127. Review.

Taylor, M.J., von Geldern, T.W., Ford, L., et al., 2019. Preclinical development of an oral anti-Wolbachia macrolide drug for the treatment of lymphatic filariasis and onchocerciasis. Sci. Transl. Med. 11, 483.

Tekle, A.H., Elhassan, E., Isiyaku, S., et al., 2012. Impact of long-term treatment of onchocerciasis with ivermectin in Kaduna State, Nigeria: first evidence of the potential for elimination in the operational area of the African Programme for Onchocerciasis Control. Parasit. Vectors. 5, 28.

Tekle, A.H., Zouré, H.G., Noma, M., et al., 2016. Progress towards onchocerciasis elimination in the participating countries of the African Programme for Onchocerciasis Control: epidemiological evaluation results. Infect. Dis. Poverty 5, 66.

Timmann, C., Abraha, R.S., Hamelmann, C., et al., 2003. Cutaneous pathology in onchocerciasis associated with pronounced systemic T-helper 2-type responses to Onchocerca volvulus. Br. J. Dermatol. 149, 782-787.

Timmann, C., van der Kamp, E., Kleensang, A., et al., 2008. Human genetic resistance to Onchocerca volvulus: evidence for linkage to chromosome $2 p$ from an autosome-wide scan. J. Infect. Dis. 198, 427433.

Tischendorf, F.W., Brattig, N.W., 1992. Cationic lysosomal proteins and reactive oxygen metabolites of eosinophilic effector cells in generalized and localized onchocerciasis. Trop. Med. Parasit. 43, 208-209.

Titanji, V.P., Nde, P.N., Ghogoinu, S.M., et al., 1996. The roles of IgG and defined antigensin cytoadherence and cytotoxicity reactions to onchocercal microfilariae. Afr. J. Health Sci. 3, 33-36.

Toé, L.D., Koala, L., Burkett-Cadena, N.D., et al., 2014. Optimization of the Esperanza window trap for the collection of the African onchocerciasis vector Simulium damnosum sensu lato. Acta Tropica 137, 393. Epub 2014/05/06. pmid:24794201.

Traore, M.O., Sarr, M.D., Badji, A., et al., 2012. Proof-of-principle of onchocerciasis elimination with ivermectin treatment in endemic foci in Africa: final results of a study in Mali and Senegal. PLoS. Negl. Trop. Dis. 6, e1825.

Traoré, S., Wilson, M.D., Sima, A., et al., 2009. The elimination of the onchocerciasis vector from the island of Bioko as a result of larviciding by the WHO African Programme for Onchocerciasis Control. Acta Trop. 111, 211-218.

Trees, A.J., Graham, S.P., Renz, A., et al., 2000. Onchocerca ochengi infections in cattle as a model for human onchocerciasis: recent developments. Parasitology 120 Suppl:S 133-142. Review. 
Trpis, M., Wergin. W.P., Murphy, C.A., 2006. Development of Onchocerca volvulus (Filarioidea: Onchocercidae) in the West African black fly Simulium yahense (Diptera: Simuliidae) in Liberia. J. Parasitol. 87: 1265-1272.

Turaga, P.S., Tierney, T.J., Bennett, K.E., et al., 2000. Immunity to onchocerciasis: cells from putatively immune individuals produce enhanced levels of interleukin-5, gamma interferon, and granulocytemacrophage colony-stimulating factor in response to Onchocerca volvulus larval and male worm antigens. Infect. Immun. 68, 1905-1911.

Unnasch, T.R., Golden, A., Cama, V., et al., 2018. Diagnostics for onchocerciasis in the era of elimination. Int. Health. 10, Suppl. 1, i20-i26.

Unnasch, T.R., Williams, S.A. 2000. The genomes of Onchocerca volvulus. Int. J. Parasitol. 30, 543-552. Review.

Vajime, C.G., Dunbar, R.W., 1975. Chromosomal identification of eight species of the subgenus Edwardsellum near and including Simulium (Edwardsellum) damnosum Theobald (Diptera: Simuliidae). Tropenmed. Parasitol. 26, 111-138.

Van Den Berghe, L., Chardome, M., Peel, E., 1964. The filarial parasite of the eastern gorilla in the Congo. J. Helminth. 38, 349-368.

Van Someren, V.D., McMahon, J., 1950. Phoretic association between Afronurus and Simulium species, and the discovery of the early stages of Simulium neavei on freshwater crabs. Nature 166, 350-351.

Van Voorhis, W.C., Hooft van Huijsduijnen, R., Wells, T.N.C. 2015. Profile of William C. Campbell, Satoshi Ōmura, and Youyou Tu, 2015 Nobel Laureates in Physiology or Medicine. Proc. Natl. Acad. Sci. U S A. $112,15773-15776$.

Verver, S., Walker, M., Kim, Y.E., et al., 2018. How can onchocerciasis elimination in Africa be accelerated? Modeling the impact of increased ivermectin treatment frequency and complementary vector control. Clin. Infect. Dis. 66 (suppl_4) S267-S274.

Vinkeles Melchers, N.V.S., Coffeng, L.E., Boussinesq, M., et al., 2020. Projected number of people with onchocerciasis-loiasis Coinfection in Africa, 1995 to 2025. Clin. Infect. Dis. 70, 2281-2289.

Vlaminck, J., Fischer, P.U., Weil, G.J., 2015. Diagnostic tools for onchocerciasis elimination programs. Trends Parasitol. 31, 571-582.

Von Geldern, T.W., Morton, H.E., Clark, R.F., et al., 2019. Discovery of ABBV-4083, a novel analog of Tylosin A that has potent anti-Wolbachia and anti-filarial activity. PLoS. Negl. Trop. Dis. 13, e0007159.

Waddy, B.B., 1969. Prospects for the control of onchocerciasis in Africa with special reference to the Volta River Basin. Bull. WHO 40, 843-858.

Wahl, G., Enyong, P., Ngosso, A., et al., 1998. Onchocerca ochengi: epidemiological evidence of crossprotection against Onchocerca volvulus in man. Parasitology 116, 349-362.

Walker, M., Specht, S., Churcher, T.S., et al., 2015. Therapeutic efficacy and macrofilaricidal activity of doxycycline for the treatment of river blindness. Clin. Infect. Dis 60, 1199- 1207.

Walker, M., Stolk, W.A., Dixon, M.A., et al., 2017. Modelling the elimination of river blindness using longterm epidemiological and programmatic data from Mali and Senegal. Epidemics 18, 4-15.

Walsh, J.F., Davies, J.B., Le Berre, R., et al., 1978. Standardization of criteria for assessing the effect of Simulium control in onchocerciasis control programmes. Trans. Roy. Soc. Trop. Med. Hyg., 72, 675676.

Walsh, J.F., Davies, J.B., Le Berre, R., 1979. Entomological aspects of the first five years of the Onchocerciasis Control Programme in the Volta River Basin. Tropenmed. Parasit. 30, 328-344.

Walsh, J.F., 1990. Review of vector control prior to the OCP. Acta Leidensia 59, 61-78.

Wanji, S., Kengne-Ouafo, J.A., Esum, M.E., et al., 2015a. Situation analysis of parasitological and entomological indices of onchocerciasis transmission in three drainage basins of the rain forest of South West Cameroon after a decade of ivermectin treatment. Parasit. Vectors, 8, 202.

Wanji, S., Kengne-Ouafo, J.A., Esum, M.E., et al., 2015b. Relationship between oral declaration on adherence to ivermectin treatment and parasitological indicators of onchocerciasis in an area of persistent transmission despite a decade of mass drug administration in Cameroon. Parasit. Vectors 8, 667.

Wegesa, P., 1970. Simulium nyasalandicum (Amani form) and S. adersi, two new potential vectors of Onchocerca volvulus in the Eastern Usambaras, North Eastern Tanzania, E. Afr. Med. J. 47, 364-367.

Weil, G.J., Steel, C., Liftis, F., et al., 2000. A rapid-format antibody card test for diagnosis of onchocerciasis. J. Infect. Dis. 183, 1796-1799.

Weller, P.F., Spencer, L.A., 2017. Functions of tissue-resident eosinophils. Nat. Rev. Immunol. 17, 746-760.

White, G.B., 1977. Man-biting species of Chrysops Meigen, Culicoides Latreille and Simulium Latreille in Ethiopia, with discussion of their vector potentialities. Trans. Roy. Soc. Trop. Med. Hyg. 71, 161-175.

WHO/ONCHO/69.75 Joint US-AID/OCCGE/WHO Technical Meeting on the Feasibility of onchocerciasis control. Tunis, 1-8 July 1968, Report.

WHO/OCP/73.1., 1973. Onchocerciasis control in the Volta River basin area. Report of the Preparatory Assistance 
ission to the Governements of Dahomey, Ghana, Ivory Coast, Mali, Niger, Togo, Upper Volta, and Annexes, PAG Report Geneva 1973. https://apps.who.int/iris/handle/10665/326101.

WHO, 1995. Onchocerciasis and its control, report of a WHO Expert Committee on Onchocerciasis Control. Geneva: World Health Organization; Technical Report Series No.: 852. https://apps.who.int/iris/handle/10665/37346.

WHO, 2002. Success in Africa: the Onchocerciasis Control Programme in West Africa, 1974-2002. WHO, Geneva.

WHO, 2017. Progress report on the elimination of human onchocerciasis, 2016-2017. Weekly epidemiological record no 45, 92, 681-700.

WHO, 2018a. Progress report on the elimination of human onchocerciasis, 2017-2018. Weekly epidemiological record no 47, 93, 633-648; https://apps.who.int/iris/bitstream/handle/10665/275983/WER9347.pdf?ua=1.

$\mathrm{WHO}, 2018 \mathrm{~b}$. Onchocerciasis elimination mapping of endemic countries is key to defeating river blindness. https://www.who.int/neglected_diseases/news/Onchocerciasis-elimination-mapping-ofendemic-countries-is-key/en/.

WHO, 2019a; https://www.who.int/news-room/fact-sheets/detail/onchocerciasis.

WHO, 2019b: https://www.who.int/neglected_diseases/news/half-million-Yemenis-treated-foronchocerciasis/en/.

WHO fact sheets, 2020: https://www.who.int/news-room/fact-sheets/detail/onchocerciasis

WHO, 2020. Onchocerciasis; https://www.who.int/onchocerciasis/en/.

Wilson, E.J., Wormall, A., 1949. Studies on suramin (Antrypol: Bayer 205). 7. Further observations on the combination of the drug with proteins. Biochem. J. 45, 224-231.

Wilson, M.D., Cheke, R.A., Flasse, S.P.J., et al., 2002. Deforestation and the spatio-temporal distribution of avannah and forest members of the Simulium damnosum complex in southern Ghana and southwestern Togo. Trans. Roy. Soc. Trop. Med. Hyg. 96, 632-639.

Yaméogo, L., Crosa, G., Samman, J., et al., 2001. Long-term assessment of insecticides treatments in West Africa: aquatic entomofauna. Chemosphere 44, 1759-1773.

Yen, J.H., 1975. Transovarial transmission of Rickettsia-like microorganisms in mosquitoes. Ann. N. Y. Acad. Sci. 266, 152-161.

Zimmerman, P.A., Dadzie, K.Y., De Sole, G., et al., 1992. Onchocerca volvulus DNA probe classification correlates with epidemiologic patterns of blindness. J. Infect. Dis. 165, 964-968.

Zarroug, I.M.A., Elaagip, A.H., Abuelmaali, S.A., et al., 2014. The impact of Merowe Dam on Simulium hamedense vector of onchocerciasis in Abu Hamed focus - Northern Sudan. Parasit. Vectors 7, 168.

Zarroug, I.M.A., Hashim, K., El Mubarak, W.A., et al., 2016. The first confirmed elimination of an onchocerciasis focus in Africa: Abu Hamed, Sudan. Am. J. Trop. Med. Hyg. 95, 1037-1040.

Zimmerman, P.A., Guderian, R.H., Aruajo, E., et al., 1994. Polymerase chain reaction-based diagnosis of Onchocerca volvulus infection: improved detection of patients with onchocerciasis. J. Infect Dis. 165, 964-968.

Zouré, H.G.M., Noma, M., Tekle, A.H. et al., 2014. The geographic distribution of onchocerciasis in the 20 participating countries of the African Programme for Onchocerciasis Control: (2) pre-control endemicity levels and estimated number infected. Parasit. Vectors 7, 326. 


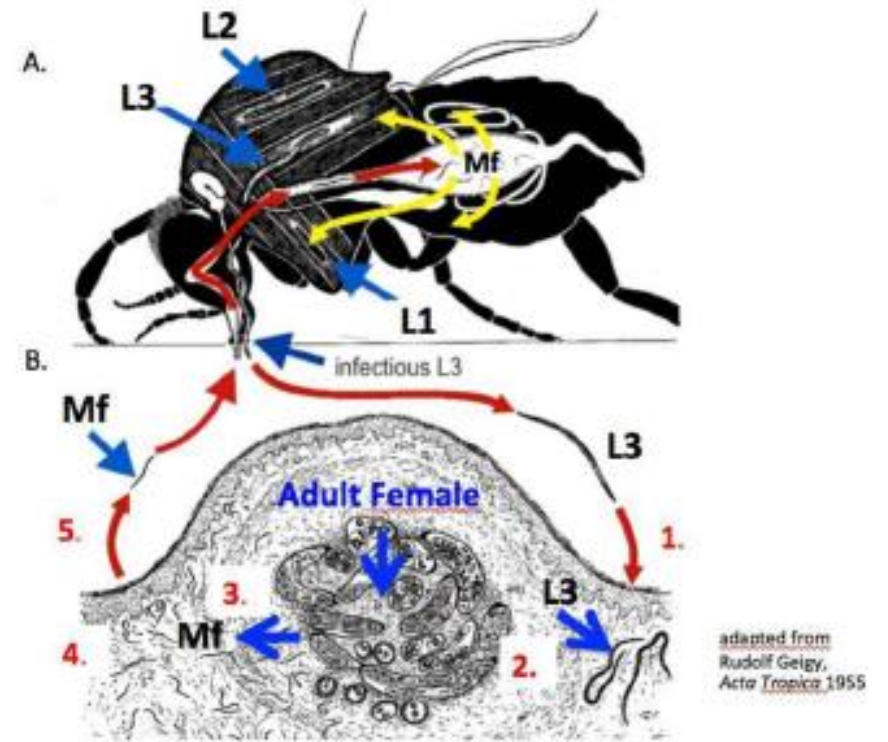

1895 Figure 1. Onchocerca volvulus life cycle: B. Infection: (1.) infective third-stage larva (L3) of O. volvulus is 1896 transmitted by the vector Simulium (A.);(2.) a histological section through an onchocercoma in the human 1897 host comprising a coiled adult female developed from a fourth-stage larva; (3.) microfilariae produced by 1898 the adult female worm which (4.) migrate from the onchocercoma into the skin (5.) to be taken up by the 1899 pool-feeding Simulium. (A.) In the vector a microfilaria develops into a first stage "sausage" form larva 1900 (L1), moults into the second-stage larva (L2) and then moults again to become a third-stage infective larva 1901 (L3) which is transmitted into the skin of the human host when the vector pool feeds (1.) (figure modified 1902 from Rudolf Geigy, Acta Tropica Supplement 6, Geigy and Herbig, Erreger und Überträger tropischer Krankheiten, 1955).

1904 


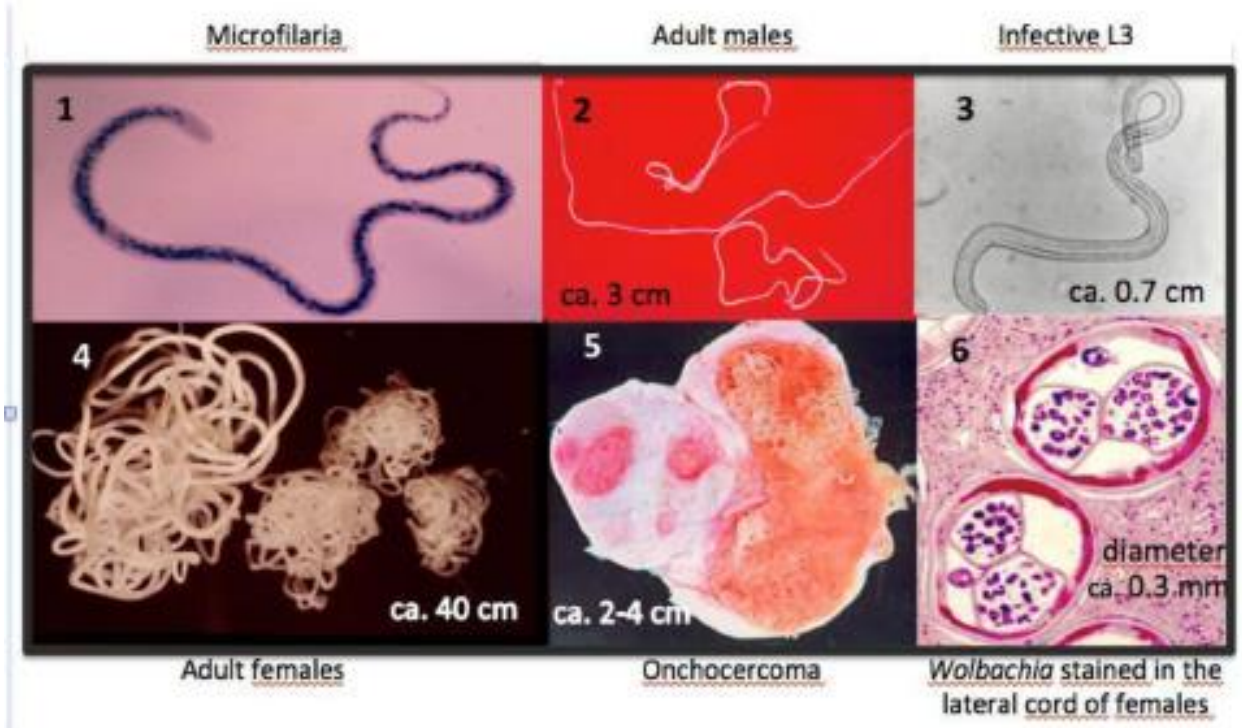

1912 Figure 2. Stages of the Onchocerca volvulus: (1.) microfilaria (about 0.2-0.3 mm long), (2.) adult males (1.5$19134.5 \mathrm{~cm})$, (3.) infective third-stage larva (0.7-0.8 mm); (4.) adult females (30-60 cm), (5.) an onchocercoma 1914 with cut adult females, (6.) sections through a nodule showing adult females (ca. $0.3 \mathrm{~mm}$ in diameter) 1915 showing two uterus branches with cut microfilariae, cut intestine and lateral cords containing 1916 endobacterial Wolbachia stained red (photo Brattig et al. 2001; D.W. Büttner, Liberia 1991; N.W. Brattig, 1917 BNITM, 2000). 


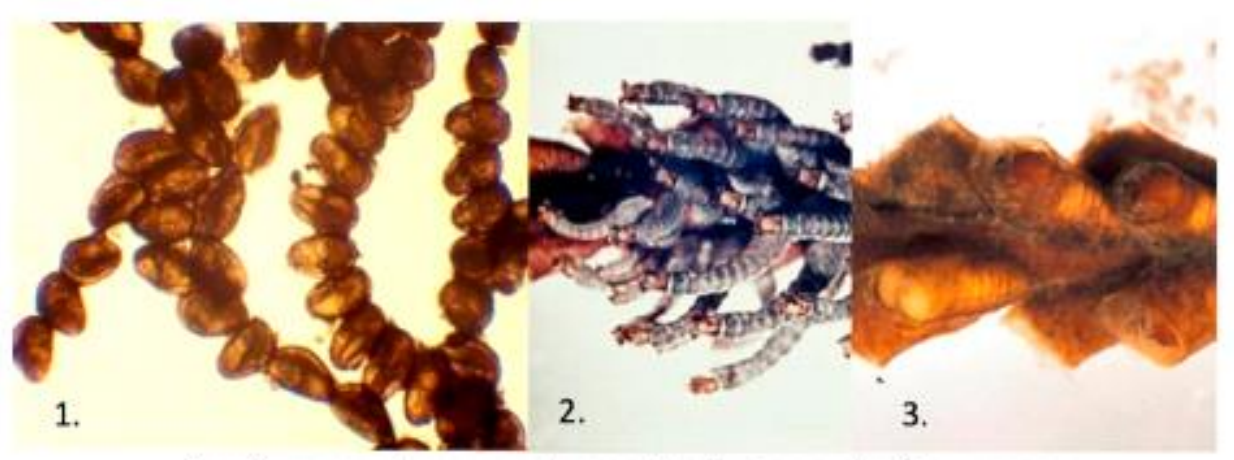

Simulium eggs, larvae and pupae developing on trailing grasses

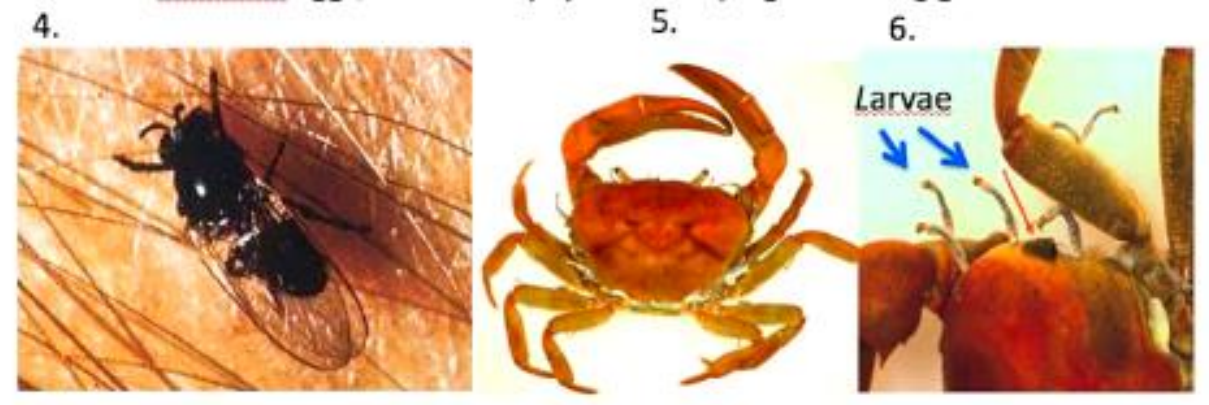

Biting adult $S$. damnosum S. neavei. larvae on phoretic crab host (Potomonautes sp.)

1921

1922

1923 Figure 3. Simulium damnosum s.l. stages: (1.) Masses of eggs Niger, Guinea, photo R. Garms, 1963), (2.)

1924 larvae on trailing vegetation and (3.) pupae, St. Paul River Liberia (photo R. Garms, 1983). (4.) Adult

1925 Simulium s.l., photo R. Garms, Liberia, 1983), (5.) Drop of blood after pool-feeding by a Simulium (photo R.

1926 Gams, Guatemala, 1974).

1927

1928 


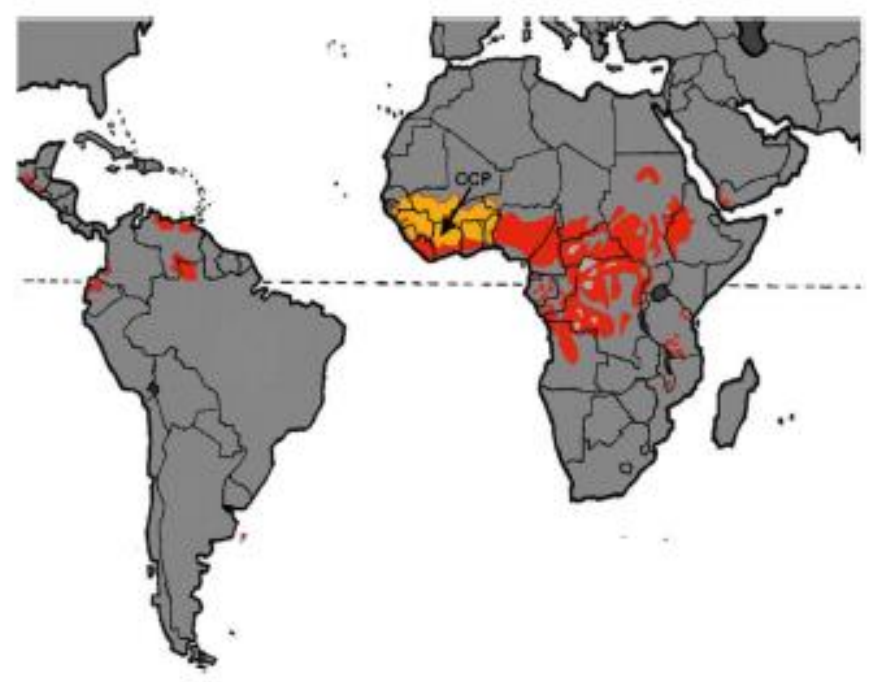

1931

1932 Figure 4. Prevalence of onchocerciasis (marked red) in 30 sub-saharan African countries, in Yemen 1933 (Arabian Peninsula) and in 6 countries of central and south America. The area of the Onchocerciasis 1934 Control Programme (1974-2002) is marked yellow (modified, WHO, 1995).

1935

1936 


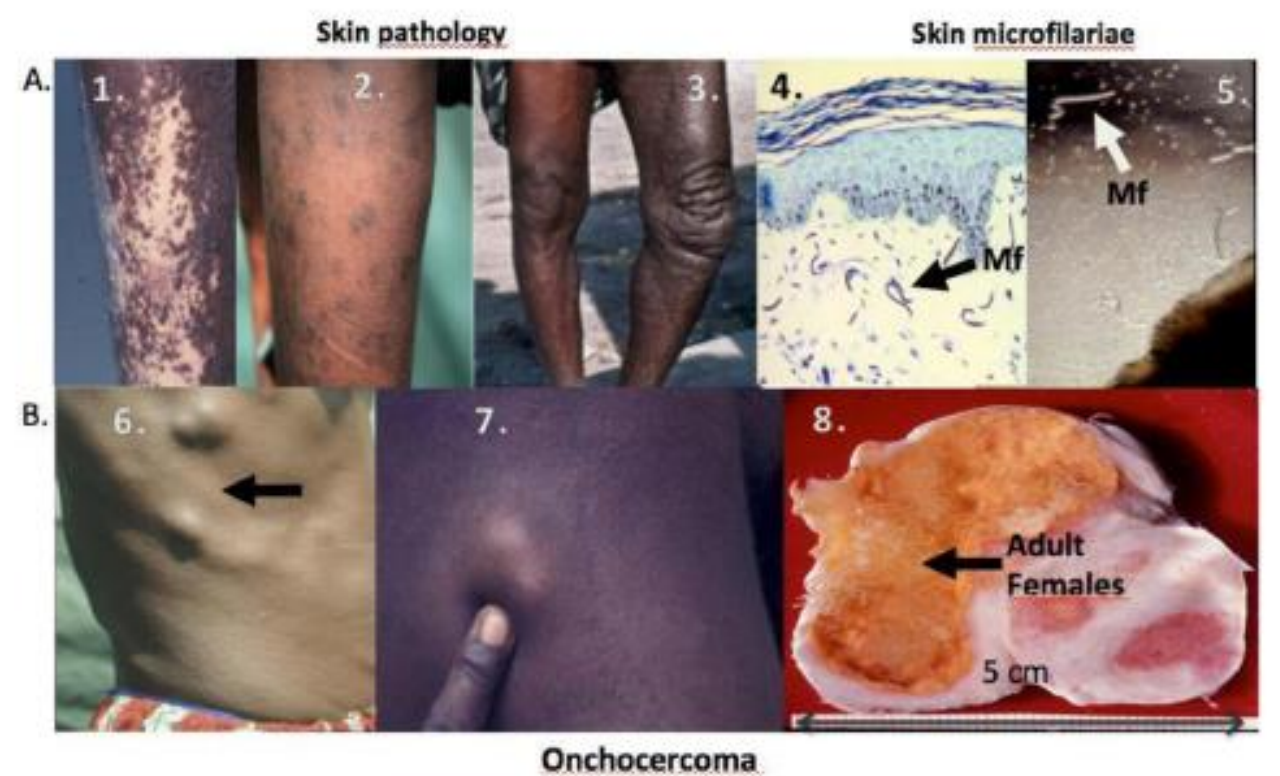

1939

1940 Figure 5. Pathology of onchocerciasis. A. Skin pathology and microfilariae: (1.) depigmentation, (2.)

1941 dermatitis, (3.) lichenification and hyperpigmentation (sowda) (Brattig, 2004; photo D.W. Büttner, Liberia

1942 1995), (4.) microfilariae in a histological section of skin (photo D.W. Büttner, Liberia, 1999), (5.)

1943 microfilaria released in vitro from a skin snip (photo N.W. Brattig, 2000); B. Onchocercomata (6.) nodules

1944 in the torso of a man (photo D.W. Büttner, Liberia, 1985), (7.) nodule in the leg of a boy (photo R. Garms,

1945 Liberia, 1971), (8.) A bisected onchocercoma with adult females (Brattig, 2004). 


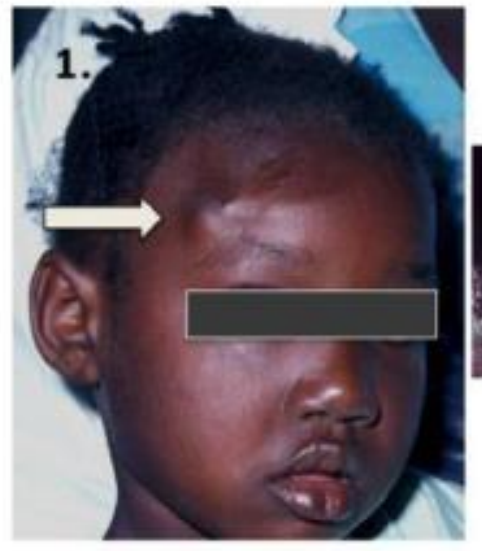

Onchocercomata in the head

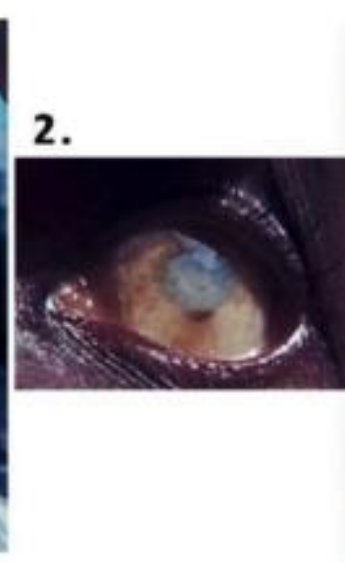

Eye with sclerosing keratitis

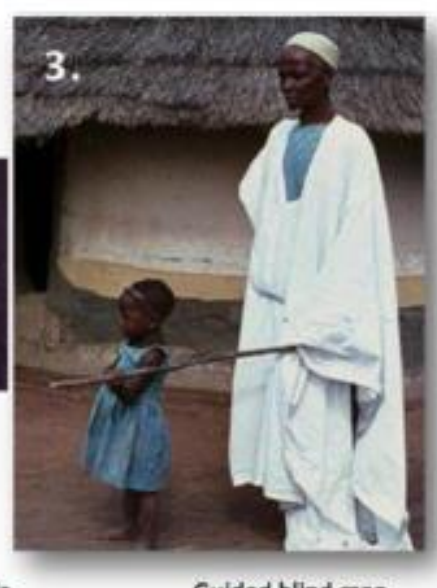

Guided blind man

1951

1952 Figure 6. Eye pathology (River blindness). (1.) Two onchocercomata in the forehead of a young child 1953 which can release microfilariae in the close proximity of the eyes (DW Büttner, Liberia,1993), (2.) cornea 1954 opacification,sclerosing keratitis (R. Garms, Kouroussa, Guinea, 1963), (3.) man blinded by onchocerciasis 1955 guided by a child (photo R. Garms, Sérékoroba Guinea, 1963). Microfilariae releasing the onchocercoma 1956 can infiltrate the eye and can lead to visual impairment and blindness. 


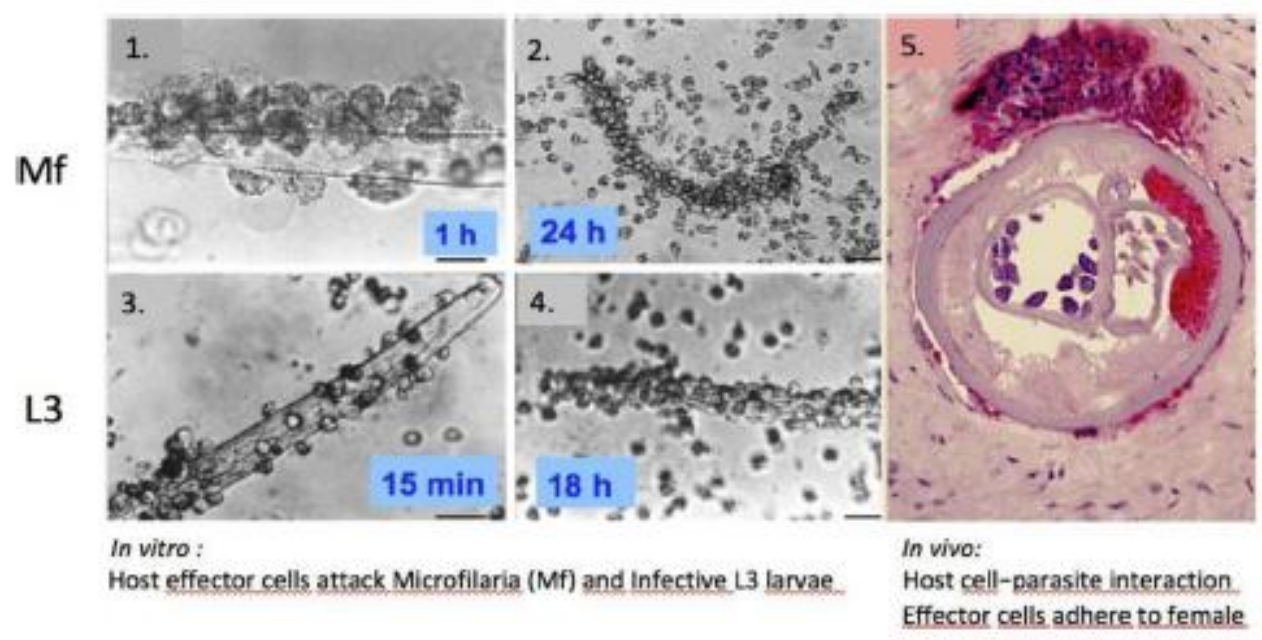

1961 Figure 7. Host-parasite interaction. (1., 2.) time-dependent in vitro attack of microfilariae (Mf) by human

1962 eosinophilic effector cells (3., 4.) and of infective $3^{\text {rd }}$ stage larvae (L3) (modified figures from Brattig,

1963 2004), (5.) Host neutrophilic granulocytes assembled at the surface of a female in a section of an

1964 onchocercoma with Wolbachia in the lateral cords of the female; the Wolbachia were stained with

1965 antibodies against Wolbachia heat shock protein 60, the neutrophils with antibodies against defensin

1966 (Brattig et al., 2001). 
1.

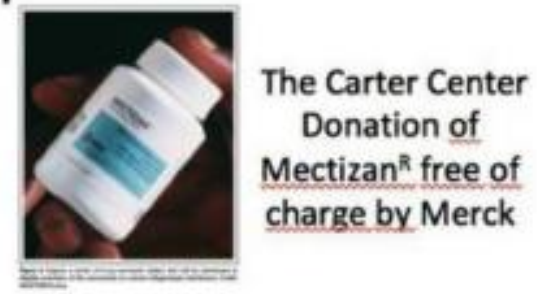

2.

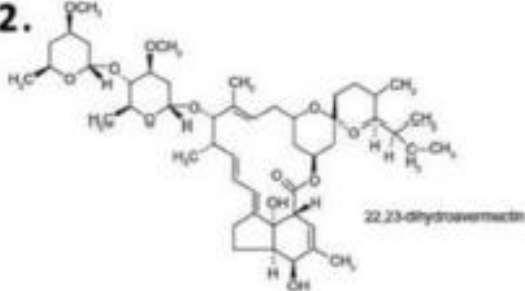

Dihydro-avermectin

Macrocyclic lactone from Streptomyces
3.

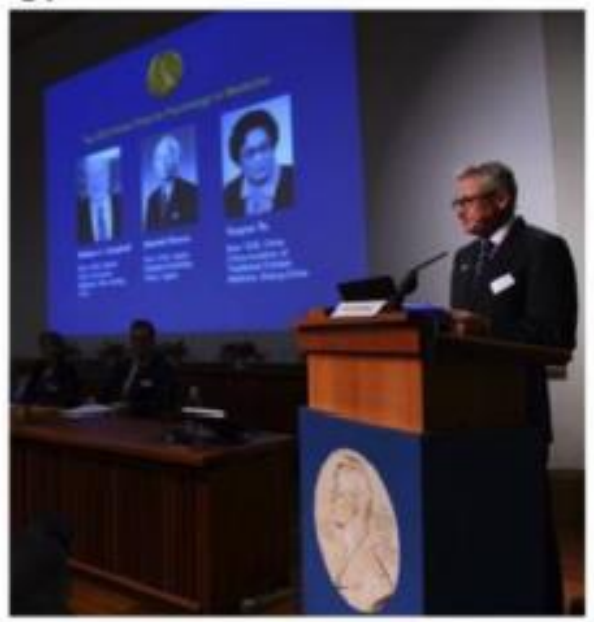

Nobel Prize in Phvsiology or Medicine, 2015

1971

1972 Figure 8. Container with Ivermectin (dihydro-avermectin), the principal drug against onchocerciasis (1.);

1973 (2) the chemical formula: a macrocyclic lactone, synthesized by Streptomyces sp., administered as

1974 Mectizan $^{R}$ (Merck Inc.) is donated free to millions of onchocerciasis patients organized by the Carter

1975 Center. (3.) In 2015, the Nobel Prize in Phyiology or Medicine was awarded at the Karolinska Institute to

1976 William C. Campbell and Satoshi Ömura for their discovery of the anti-filarial compound Ivermectin.

1977

1978

1979 


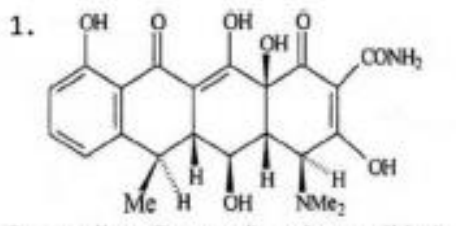

Doxvcycline: Tetracycline-class antiblotic

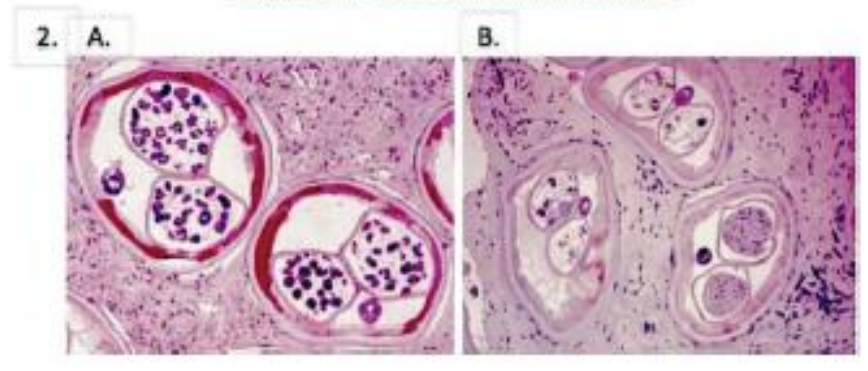

1981

1982 Figure 9. Antibacterial doxycyline, (1.) a tetracycline-class polycyclic antibiotic agent kills Wolbachia 1983 endobacteriae in Onchocerca microfilariae and is introduced for treatment of onchocerciasis in 2003 1984 (Hoerauf et al., 2001; Walker et al., 2015). (2.) Sections through onchocercomata (A.) from an untreated 1985 patient which comprise Wolbachia stained red in the cords and (B.) females in an onchocercoma from 1986 doxycycline-treated patients with depleted Wolbachia (Brattig et al., 2001).

1987

1988

1989 
1.

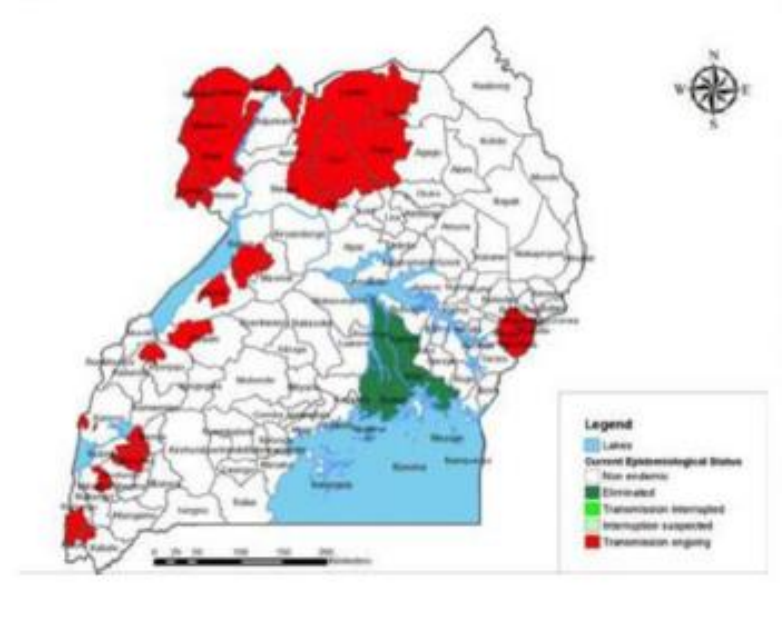

2.

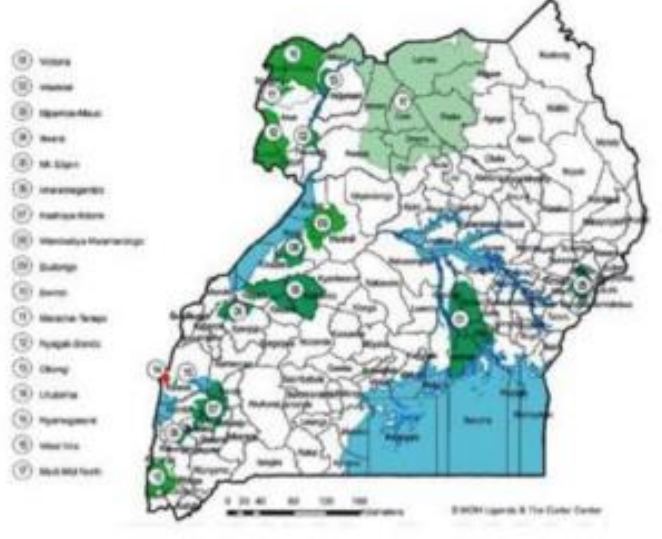

1993 Figure 10. Progress of onchocerciasis elimination in Uganda by 2019. Comparison of transmission in 1992 1994 (1.) and in 2019 (2.) In 1992 (1.), only the historical large Victoria Nile focus (green) had been eliminated 1995 after intensive vector control activities. In 2019 (2.), onchocerciasis had been very successfully eliminated 1996 from 15 (green) of the original 17 foci, transmission was possible still ongoing in the Madi Mid North focus 1997 (focus 17; light green) and still active in the small Lhubiriha focus on the border with D.R. Congo (focus 14; 1998 red) (copyright MOH Uganda and The Carter Center; 2019 kindly provided by Moses Katabarwa, 2020). 1999 